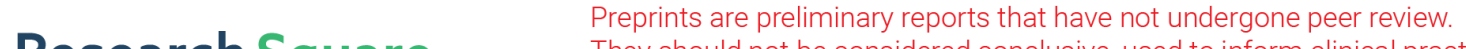 They should not be considered conclusive, used to inform clinical practice, or referenced by the media as validated information. \\ Determining the three-dimensional atomic structure of a metallic glass
}

\section{Yao Yang}

University of California Los Angeles https://orcid.org/0000-0002-2896-9046

Jihan Zhou

University of California, Los Angeles

\section{Fan Zhu}

University of California, Los Angeles

\section{Yakun Yuan}

University of California Los Angeles

\section{Dillan Chang}

University of California Los Angeles https://orcid.org/0000-0002-3715-1596

\section{Dennis Kim}

MIT https://orcid.org/0000-0002-5707-2609

\section{Minh Pham}

University of California Los Angeles

\section{Arjun Rana}

University of California Los Angeles

\section{Xuezeng Tian}

University of California Los Angeles https://orcid.org/0000-0002-4563-1960

\section{Yonggang Yao}

University of Maryland https://orcid.org/0000-0002-9191-2982

\section{Stanley Osher}

University of California Los Angeles

\section{Andreas Schmid}

NCEM, Molecular Foundry, Lawrence Berkeley National Laboratory

\section{Liangbing $\mathrm{Hu}$}

University of Maryland at College Park https://orcid.org/0000-0002-9456-9315

\section{Peter Ercius}

Lawrence Berkeley National Laboratory https://orcid.org/0000-0002-6762-9976

Jianwei (John) Miao ( $\sim$ miao@physics.ucla.edu )

University of California Los Angeles https://orcid.org/0000-0003-4033-3945 
Keywords: glass, three-dimensional (3D) atomic structure, amorphous solids, atomic electron tomography Posted Date: October 16th, 2020

DOI: https://doi.org/10.21203/rs.3.rs-88474/v1

License: (c) (1) This work is licensed under a Creative Commons Attribution 4.0 International License. Read Full License 
1 Determining the three-dimensional atomic structure of a

2 metallic glass

3 Yao Yang ${ }^{1 *}$, Jihan Zhou ${ }^{*}$, Fan Zhu ${ }^{1 *}$, Yakun Yuan ${ }^{1 *}$, Dillan J. Chang ${ }^{1}$, Dennis S. Kim ${ }^{1}$,

4 Minh Pham ${ }^{2}$, Arjun Rana ${ }^{1}$, Xuezeng Tian ${ }^{1}$, Yonggang Yao ${ }^{3}$, Stanley J. Osher ${ }^{2}$, Andreas

$5 \quad$ K. Schmid ${ }^{4}$, Liangbing $\mathrm{Hu}^{3}$, Peter Ercius ${ }^{4} \&$ Jianwei Miao $^{1}$

$6 \quad{ }^{1}$ Department of Physics \& Astronomy, STROBE NSF Science \& Technology Center and

7 California NanoSystems Institute, University of California, Los Angeles, CA 90095, USA.

$8{ }^{2}$ Department of Mathematics, University of California, Los Angeles, CA 90095, USA.

$9 \quad{ }^{3}$ Department of Materials Science and Engineering, University of Maryland, College

10 Park, Maryland, 20742, USA. ${ }^{4}$ National Center for Electron Microscopy, Molecular

11 Foundry, Lawrence Berkeley National Laboratory, Berkeley, CA 94720, USA.

12 *These authors contributed equally to this work.

13 Amorphous solids such as glass are ubiquitous in our daily life and have found broad applications ranging from window glass and solar cells to telecommunications and transformer cores ${ }^{1,2}$. However, due to the lack of long-range order, the threedimensional (3D) atomic structure of amorphous solids have thus far defied any

17 direct experimental determination without model fitting ${ }^{3-13}$. Here, using a multicomponent metallic glass as a proof-of-principle, we advance atomic electron tomography to determine the 3D atomic positions in an amorphous solid for the first time. We quantitatively characterize the short-range order (SRO) and mediumrange order (MRO) of the 3D atomic arrangement. We find that although the 3D atomic packing of the SRO is geometrically disordered, some SRO connect with each other to form crystal-like networks and give rise to MRO. We identify four crystal- 
cubic and simple cubic - coexisting in the sample, which show translational but no orientational order. These observations confirm that the 3D atomic structure in some parts of the sample is consistent with the efficient cluster packing model ${ }^{8,10-}$ ${ }^{12,20}$. Looking forward, we anticipate this experiment will open the door to determining the 3D atomic coordinates of various amorphous solids, whose impact on non-crystalline solids may be comparable to the first $3 \mathrm{D}$ crystal structure solved by $x$-ray crystallography over a century ago $^{14}$.

Since the first discovery in $1960^{15}$, metallic glasses have been actively studied for fundamental interest and practical applications ${ }^{7-12,16-20}$. However, due to their disordered structure, the 3D atomic arrangement of metallic glasses cannot be determined by crystallography ${ }^{21}$. Over the years, a number of experimental and computational methods have been used to study the metallic glass structure, such as x-ray/neutron diffraction ${ }^{22,23}$, x-ray absorption fine structure ${ }^{9}$, high-resolution transmission electron microscopy ${ }^{24}$, fluctuation electron microscopy ${ }^{25}$, angstrom- and nano-beam electron diffraction ${ }^{13,26,27}$, nuclear magnetic resonance ${ }^{28}$, density functional theory ${ }^{29}$, molecular dynamics simulations ${ }^{30-33}$ and reverse Monte Carlo modelling ${ }^{9,25}$. Despite all these developments, however, there was no experimental method available to directly determine all the $3 \mathrm{D}$ atomic positions in metallic glass samples. One experimental method that can potentially solve this long-standing problem is atomic electron tomography $(\mathrm{AET})^{34,35}$. AET combines high-resolution tomographic tilt series with advanced iterative algorithms to resolve the $3 \mathrm{D}$ atomic structure of materials without assuming crystallinity, which has been applied to image grain boundaries, anti-phase boundaries, stacking faults, dislocations, point defects, chemical order/disorder, atomic-scale ripples, bond distortion and strain tensors with unprecedented 3D detail ${ }^{36-41}$. More recently, 4D (3D + time) AET 
has been developed to observe crystal nucleation at atomic resolution, showing that early stage nucleation results are inconsistent with classical nucleation theory ${ }^{42}$. Here, we use a multi-component metallic glass as a model and advance AET to determine its 3D atomic positions with a precision of 21 picometers.

\section{Determining the 3D atomic positions in a multi-component metallic glass}

54 The samples were synthesized by a carbothermal shock technique with a high cooling rate (Extended Data Fig. 1a, Supplementary video 1 and Methods), which created high entropy alloy nanoparticles with multi-metal components ${ }^{43}$. The energy-dispersive X-ray spectroscopy data show the nanoparticles are composed of eight elements: $\mathrm{Co}, \mathrm{Ni}, \mathrm{Ru}$, Rh, Pd, Ag, Ir and Pt (Extended Data Fig. 1b-k). Tomographic tilt series were acquired from seven nanoparticles using a scanning transmission electron microscope with an annular dark-field detector (Extended Data Table 1). While most of the nanoparticles are crystalline or polycrystalline, particles 1 and 2 have disordered structure (Extended Data

Fig. 2). In this study, we focus on the more disordered nanoparticle (particle 1), from which a tilt series of 55 images were measured (Fig. 1a and Extended Data Fig. 3a). Although some crystalline features are present in several images, the diffraction patterns calculated from the images show the amorphous halo. From the average diffraction pattern (Fig. 1b), we derived the radial distribution function (RDF) (Extended Data Fig. 3c), exhibiting the amorphous structure of the nanoparticle. After pre-processing and image denoising, the tilt series was reconstructed and the 3D atomic positions were traced and classified (Fig. 1c, d, Supplementary Video 2 and Methods). Since the image contrast in the $3 \mathrm{D}$ reconstruction depends on the atomic number ${ }^{40-42}$, presently AET is only sensitive enough to classify the eight elements into three different types: $\mathrm{Co}$ and $\mathrm{Ni}$ as 
obtained the 3D atomic model of the nanoparticle, consisting of 8322, 6896 and 3138 atoms for type 1, 2 and 3, respectively. To verify the reconstruction, atom tracing and classification procedure, we calculated 55 images from the experimental atomic model using multislice simulations (Methods). Extended Data Fig. 4c and d shows the consistency between the experimental and calculated images. We then applied the reconstruction, atom tracing and classification procedure to obtain a new $3 \mathrm{D}$ atomic model from the 55 multislice images. By comparing the two models, we estimated that $97.37 \%$ of atoms were correctly identified with a 3D precision of $21 \mathrm{pm}$ (Methods and Extended Data Fig. 4e).

Figure 1e and Supplementary video 3 show the experimental 3D atomic model of the nanoparticle with type 1, 2 and 3 atoms in green, blue and red, respectively. To quantitatively characterize the atomic structure, we employed the local bond orientational order (BOO) parameters to distinguish between the disordered, face-centred cubic (fcc), hexagonal close-packed (hcp) and body-centered cubic (bcc) structures (Methods). Figure 1f shows the local BOO parameters of all the atoms in the nanoparticle, indicating the majority of atoms severely deviate from the fcc, hep and bcc crystal structures. For a comparison, the local BOO parameters of the seven nanoparticles are shown in Extended Data Fig. 2h-n. To separate crystal nuclei from the amorphous structure, we used the normalized BOO parameter to identify the crystal nuclei (Methods). By choosing the criterion of the normalized BOO parameter $\geq 0.5$ (Extended Data Fig. 2o), we identified $15.46 \%$ of the total atoms forming crystal nuclei in the nanoparticle (Extended Data Fig5a), which contribute to the crystalline features observed in several images (Extended Data Fig. 3a). In the following sections, we focus on the analysis of the atoms with the normalized BOO parameter $<0.5$. 
Figure $1 \mathrm{~g}$ shows the RDF of the amorphous structure of the 3D atomic model (Methods), where the weak second-peak splitting is consistent with previous observation in high entropy bulk metallic glasses ${ }^{44}$. The ratios of the second, third, fourth and fifth to the first peak position are $1.74,1.99,2.64$ and 3.51, respectively, which agree with those of metallic glasses ${ }^{45,46}$. The partial pair distribution functions (PDFs) between type 1, 2 and 3 atoms are shown in Fig. 1h. By fitting a Gaussian to the first peaks in the partial PDFs, we determined the type 11, 12, 13, 22, 23 and 33 bond lengths to be $2.59,2.71$, 2.78, 2.72, 2.75 and $2.9 \AA$, respectively. In particular, the partial PDF for the type 33 atoms (the yellow curve) exhibits a unique feature with the second peak higher than the first peak, indicating that the majority of type 3 atoms are distributed beyond the SRO.

\section{The short-range order}

To determine the SRO in the metallic glass sample, we used the Voronoi tessellation to characterize the local atomic arrangement ${ }^{6}$. This method identifies the nearest neighbour atoms around each central atom to form a Voronoi polyhedron, which is designated by a Voronoi index $<n_{3}, n_{4}, n_{5}, n_{6}>$ with $n_{i}$ denoting the number of $i$-edged faces. Figure $2 \mathrm{a}$ shows the ten most abundant Voronoi polyhedra in the nanoparticle with a fraction ranging from $5.02 \%$ to $1.72 \%$, most of which are geometrically disordered and commonly observed in model metallic glasses ${ }^{11}$ such as $\left.\left.\langle 0,4,4,3\rangle,<0,3,6,3\right\rangle,<0,4,4,2\right\rangle$ and $<0,3,6,2>$ (Fig. 2b). The small fractions of the Voronoi polyhedra suggest that the sample is not a well relaxed metallic glass due to its poor glass forming ability ${ }^{18}$. Figure $2 \mathrm{c}$ shows the local symmetry distribution of all the faces of the Voronoi polyhedra. The 3-, 4-, 5and 6-edged faces account for $3.27 \%, 29.14 \%, 43.91 \%$ and $23.67 \%$, respectively, revealing that 5 -edged faces are most abundant in the SRO. But only $7.03 \%$ of all the Voronoi polyhedra are distorted icosahedra, including Voronoi indices $<0,0,12,0>$, 
$121<0,1,10,2>,<0,2,8,2>$ and $<0,2,8,1>$. This observation indicates that most 5-edged faces

122 do not form distorted icosahedra in this metallic glass nanoparticle. From the Voronoi

123 tessellation, we also calculated the distribution of the coordination number (CN) (Fig. $2 \mathrm{~d}$

124 and Methods), where the average CNs for types 1, 2 and 3 atoms are 11.97, 12.02 and

125 12.41, respectively. Based on the partial CNs (Extended Data Fig. 5b), we quantified the 126 chemical SRO using the Warren-Cowley parameters (Methods), indicating that the type 12711 and 23 bonds are favoured, but the type 12 and 33 bonds are unfavoured. These results 128 are consistent with the observations of the shortening of the type 11 and 23 bonds and the 129 lengthening of the type 12 and 33 bonds (Methods).

\section{The medium-range order}

131 From the partial PDF of type 33 atoms (Fig. 1h, the yellow curve), we observed that the 132 highest peak is located at $4.77 \AA$ and 1.49 times higher than the nearest neighbour peak.

133 This result indicates that the majority of type 3 atoms are distributed in the second 134 coordination shell, which is between the first $(3.86 \AA)$ and the second minimum $(6.08 \AA)$

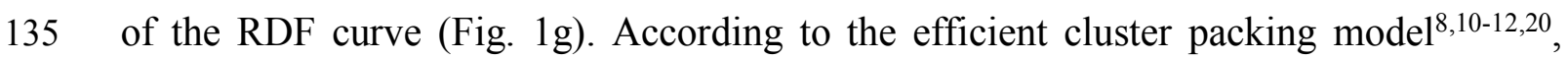
136 solute atoms are surrounded by solvent atoms to form solute-centred clusters. These 137 solute-centred clusters act as the basic building blocks and are densely packed in 3D space 138 to constitute the MRO of metallic glasses. To quantitatively test this model with experimental data, we examined the distribution of the type 3 atoms in the second 140 coordination shell and identified $85.47 \%$ of type 3 atoms as solute centres (Extended Data 141 Fig. 5c and Methods). These solute centres are surrounded mainly by type 1 and 2 solvent atoms to form atomic clusters. Extended Data Fig. 5d shows the ten most abundant Voronoi polyhedra of the solute-centred clusters. The solute-centred clusters connect with each other by sharing one (a vertex), two (an edge) and three atoms (a face) as well as 
protrude into each other by sharing four and five atoms (Fig. 3a-e). Figure $3 \mathrm{f}$ shows the statistical distribution of the number of the solute-centred cluster pairs, which share from one to five atoms.

To locate the MRO in the metallic glass nanoparticle, we implemented a breadthfirst search algorithm to look for the fcc-, hcp-, bcc-, simple cubic (sc-) and icosahedrallike structures of the solute centres (Methods). This algorithm globally searches for the MRO with a maximum number of solute centres. Each MRO is defined to have five or more solute centres with each solute centre falling within a $0.75 \AA$ radius to the fcc, hcp, bcc, sc lattice or icosahedral vertices. We found that four types of MRO (fcc-, hcp-, bccand sc-like) coexist in the sample (Methods). Although we did not observe icosahedrallike MRO in this sample, our work does not rule out its existence in other metallic glasses $^{9}$. Figure $3 \mathrm{~g}$ shows the histogram of the four types of MRO as a function of the size (i.e. the number of solute centres), where the inset illustrates the fraction of the solute centre atoms in the four types of MRO. Figure $3 \mathrm{~h}$ and Supplementary Video 4 show the 3D distribution of the MRO with each having eight solute centres or more. To verify our analysis, we also searched for the MRO with a $1 \AA$ and $0.5 \AA$ radius cut-off and observed the coexistence of the four types of MRO with different cut-off radii (Extended Data Figs. 6 and 7).

Next, we quantitatively characterized the MRO with a $0.75 \AA$ radius cut-off. Figure $4 \mathrm{a}$ and $\mathrm{b}$ shows the length and volume distribution of the MRO in the metallic glass nanoparticle. The average length and volume of the fcc-, hcp-, bcc- and sc-like MRO were measured to be $2.27 \pm 0.50,2.40 \pm 0.42,2.07 \pm 0.38,2.11 \pm 0.48 \mathrm{~nm}, 1.80 \pm 0.64$, $1.96 \pm 0.53,1.63 \pm 0.46$ and $1.96 \pm 0.74 \mathrm{~nm}^{3}$, respectively. Figure 5a, c, e and g shows four representative fcc-, hcp-, bcc- and sc-like MRO, where the solute-centred clusters 
exhibit only translational but no orientational order. To better visualize these MRO, the solute centres are orientated along the fcc, hcp, bcc and sc zone axes (Fig. 5b, d, f and h), showing that the $3 \mathrm{D}$ shapes of the MRO are anisotropic and the networks are distorted. We calculated the partial PDFs of all the fcc-, hcp-, bcc- and sc-like solute centres in the metallic glass nanoparticle and their corresponding maximum peak positions are at 4.62, 4.77, 4.82 and $3.88 \AA$, respectively (Fig. 4c). These peak positions represent the average nearest neighbour distances of the solute centres in the four crystal-like MRO and the broadened peaks signify the severe deviation of the MRO from perfect crystal lattices. Compared with the other three partial PDFs, the partial PDF of the sc-like MRO has two peaks and the ratio of the second to the first peak position is about $\sqrt{2}$ (Fig. 4c, the purple curve), which corresponds to the ratio of the diagonal to the side length of a square. The shorter nearest neighbour distance of the sc-like MRO than the other three crystal-like networks and the appearance of the two peaks in the partial PDF indicate that the sc-like solute-centred clusters are more closely connected with their neighbours. Figure 4d shows the distribution of sharing one, two, three, four and five atoms between neighbouring solute-centred clusters for the four types of the MRO, confirming that the solute-centred clusters in sc-like MRO tend to share more atoms with their neighbours than those in other types of MRO.

Our quantitative analysis of the SRO and MRO in a multi-component metallic glass provides direct experimental evidence to support the general framework of the efficient cluster packing model ${ }^{8,10-12,20}$, that is, solute-centred clusters are densely packed in some parts of the sample to give rise to the MRO. We observed the chemical SRO, the bond shortening and lengthening, and the coexistence of fcc-, hcp-, bcc- and sc-like MRO in the multi-component metallic glass. By quantifying their length, volume and 3D 
structure, we found that the MRO not only has a large variation in length and volume, but also significantly deviates from perfect crystal lattices (Fig. 4c). As the size of MRO is comparable to that of shear transformation zones in metallic glasses ${ }^{11,20,47,48}$, we expect that AET could also be applied to determine the 3D atomic structure of shear transformation zones and link the structure and properties of metallic glasses ${ }^{11}$.

\section{Outlook}

Over the last century, crystallography has been broadly applied to determine the 3D atomic structure of crystalline samples ${ }^{21}$. The quantitative 3D structural information has been fundamental to the development of many scientific fields. However, for amorphous solids, their 3D structure has been primarily inferred from experimental data, where either the average statistical structural information can be obtained or model fitting is required to analyse the local atomic order ${ }^{8-13}$. These qualitative approaches have hindered our fundamental understanding of the 3D structure of amorphous solids and related phenomena such as the crystal-amorphous phase transition and the glass transition ${ }^{11,49,50}$. Here, we demonstrate the ability to directly determine the $3 \mathrm{D}$ atomic structure of an amorphous solid using AET, which enables us to quantitatively analyse the SRO and MRO at the single-atom level. Although we focus on a metallic glass nanoparticle in this study, this method is generally applicable to different sample geometries such as thin films and extended objects (Extended Data Figs. 8 and 9, Methods). Thus, we expect that this work could open a new era in determining the 3D structure of a wide range of amorphous solids.

\section{References}

1. Zallen, R. The Physics of Amorphous Solids (Wiley, 1998).

2. Elliott, S. R. Physics of Amorphous Materials (Harlow, Essex, England: Longman 
Scientific \& Technical, 1990).

3. Zachariasen, W. H. The atomic arrangement in glass. J. Am. Chem. Soc. 54, 3841-3851 (1932).

4. Bernal, J. D. \& Mason, J. Packing of spheres: co-ordination of randomly packed spheres. Nature 188, 910-911 (1960).

5. Scott, G. D. Packing of equal spheres. Nature 188, 908-909 (1960).

6. Finney, J. L. Random packings and structure of simple liquids. 1. Geometry of random close packing. Proc. Roy Soc. Lond. Ser. A - Math. Phys. Sci. 319,479-493 (1970).

7. Nelson, D. R. \& Spaepen, F. Polytetrahedral order in condensed matter. Solid State Phys. 42, 1-90 (1989).

8. Miracle, D. B. A structural model for metallic glasses. Nat. Mater. 3, 697-702 (2004).

9. Sheng, H. W., Luo, W. K., Alamgir, F. M., Bai, J. M. \& Ma, E. Atomic packing and short-to-medium-range order in metallic glasses. Nature 439, 419-425 (2006).

10. Miracle, D. B., Egami, T., Flores, K. M. \& Kelton, K. F. Structural aspects of metallic glasses. MRS Bull. 32, 629-634 (2007).

11. Cheng, Y. Q. \& Ma, E. Atomic-level structure and structure-property relationship in metallic glasses. Prog. Mater. Sci. 56, 379-473 (2011).

12. Miracle, D. B. A Physical Model for Metallic Glass Structures: An Introduction and Update. JOM 64, 846-855(2012).

13. Hirata, A.et al. Geometric frustration of icosahedron in metallic glasses. Science 341, 376-379 (2013).

14. Bragg, W. L. The structure of some crystals as indicated by their diffraction of X-rays. Proc. R. Soc. London Ser. A 89, 248-277 (1913).

15. Klement Jun, W., Willens, R. H. \& Duwez, P. Non-crystalline structure in solidified gold-silicon alloys. Nature 187, 869-870 (1960).

16. Greer, A. L. Metallic glasses. Science 267, 1947-1953 (1995).

17. Peker, A. \& Johnson, W. L. A highly processable metallic glass: $\mathrm{Zr}_{41.2} \mathrm{Ti}_{13.8} \mathrm{Cu}_{12.5} \mathrm{Ni}_{10}$. 
${ }_{0} \mathrm{Be}_{22.5 .}$. Appl. Phys. Lett. 63, 2342-2344 (1993).

18. Inoue, A. Stabilization of metallic supercooled liquid and bulk amorphous alloys. Acta Mater. 48, 279-306 (2000).

19. Wang, W. H., Dong, C. \& Shek. C. H. Bulk metallic glasses. Mater. Sci. Eng.: R: Rep. 44, 45-89 (2004).

20. Chen, M.W. A brief overview of bulk metallic glasses. NPG Asia Mater. 3, 82-90 (2011).

21. Crystallography at 100. Science 343, 1091-1116 (2014).

22. Egami, T. \& Billinge, S. J. L. Underneath the Bragg Peaks: Structural Analysis of Complex Materials. (Pergamon Materials Series, v.7, 2003).

23. Kelton, K. F. et al. First X-ray scattering studies on electrostatically levitated metallic liquids: demonstrated influence of local icosahedral order on the nucleation barrier. Phys. Rev. Lett. 90, 195504 (2003).

24. Zhong, L., Wang, J., Sheng, H., Zhang, Z. \& Mao, S. X. Formation of monatomic metallic glasses through ultrafast liquid quenching. Nature 512, 177-180 (2014).

25. Hwang, J. et al. Nanoscale structure and structural relaxation in $\mathrm{Zr}_{50} \mathrm{Cu}_{45} \mathrm{~A}_{15}$ bulk metallic glass. Phys. Rev. Lett. 108, 195505 (2012).

26. Hirata, A. et al. Direct observation of local atomic order in a metallic glass. Nat. Mater. 10, 28-33 (2011).

27. Pekin, T.C. et al. Direct measurement of nanostructural change during in situ deformation of a bulk metallic glass. Nat. Commun. 10, 2445 (2019).

28. Tang, X. P., Geyer, U., Busch, R., Johnson, W. L. \& Wu, Y. Diffusion mechanisms in metallic supercooled liquids and glasses. Nature 402, 160-162 (1999).

29. Sachdev, S. \& Nelson, D. R. Order in metallic glasses and icosahedral crystals. Phys. Rev. B 32, 4592-4606 (1985).

30. Tang, C., \& Harrowell, P. Anomalously slow crystal growth of the glass-forming alloy CuZr. Nat. Mater. 12, 507-511 (2013). 
31. Cheng, Y. Q., Ma, E. \& Sheng, H. W. Atomic level structure in multicomponent bulk metallic glass. Phys. Rev. Lett. 102, 245501 (2009).

32. Hu, Y. C., Li, F. X., Li, M. Z., Bai, H. Y. \& Wang, W. H. Five-fold symmetry as indicator of dynamic arrest in metallic glass-forming liquids. Nat. Commun. 6, 8310 (2015).

33. Ding, J. \& Ma, E. Computational modeling sheds light on structural evolution in metallic glasses and supercooled liquids. Npj Comput. Mater. 3, 9 (2017).

34. Scott, M. C. et al. Electron tomography at 2.4-angstrom resolution. Nature 483, 444447 (2012).

35. Miao, J., Ercius, P. \& Billinge, S. J. L. Atomic electron tomography: 3D structures without crystals. Science 353, aaf2157 (2016).

36. Chen, C.-C. et al. Three-dimensional imaging of dislocations in a nanoparticle at atomic resolution. Nature 496, 74-77 (2013).

37. Goris, B. et al. Three-Dimensional Elemental Mapping at the Atomic Scale in Bimetallic Nanocrystals. Nano Lett. 13, 4236-4241 (2013).

38. Xu, R. et al. Three-dimensional coordinates of individual atoms in materials revealed by electron tomography. Nat. Mater. 14, 1099-1103 (2015).

39. Haberfehlner, G. et al. Formation of bimetallic clusters in superfluid helium nanodroplets analysed by atomic resolution electron tomography. Nat. Commun. 6, 8779 (2015).

40. Yang, Y. et al. Deciphering chemical order/disorder and material properties at the single-atom level. Nature 542, 75-79 (2017).

41. Tian, X. et al. Correlating the three-dimensional atomic defects and electronic properties of two-dimensional transition metal dichalcogenides. Nat. Mater. 19, 867873 (2020).

42. Zhou, J. et al. Observing crystal nucleation in four dimensions using atomic electron tomography. Nature 570, 500-503 (2019). 
43. Yao, Y. et al. Carbothermal shock synthesis of high-entropy-alloy nanoparticles. Science 359, $1489-1494$ (2018).

44. Kim, J. Y. et al. Utilization of high entropy alloy characteristics in Er-Gd-Y-Al-Co high entropy bulk metallic glass. Acta Mater. 155, 350-361 (2018).

45. Liu, X. J. et al. Metallic liquids and glasses: atomic order and global packing. Phys. Rev. Lett. 105, 155501 (2010).

46. Wu, Z. W., Li, M. Z., Wang, W. H. Liu, K.X. Hidden topological order and its correlation with glass-forming ability in metallic glasses. Nat. Commun. 6, 6035 (2015).

47. Spaepen, F. A microscopic mechanism for steady state inhomogeneous flow in metallic glasses. Acta Metall. 25, 407-415 (1977).

48. Argon A. S. Plastic-deformation in metallic glasses. Acta Metall. 27, 47-58 (1979).

49. Johnson, W. L. Thermodynamic and kinetic aspects of the crystal to glass transformation in metallic materials. Prog. Mater. Sci. 30, 81-134 (1986).

50. Debenedetti, P. G. \& Stillinger, F. H. Supercooled liquids and the glass transition. Nature 410, 259-267 (2001).

Acknowledgements We thank J. Ding for stimulating discussions, H. B. Yu for providing a molecular dynamics simulated atomic model of the $\mathrm{Cu}_{65} \mathrm{Zr}_{35}$ metallic glass, and D. J. Kline and M. R. Zachariah for assistance with the temperature measurements. This work was primarily supported by STROBE: A National Science Foundation Science \& Technology Center under Grant No. DMR 1548924. This work was also supported by the U.S. Department of Energy, Office of Science, Basic Energy Sciences, Division of Materials Sciences and Engineering under Award No. DE-SC0010378 (3D image reconstruction, atom tracing and classification, and multislice simulations) and the NSF DMREF program under Award No. DMR-1437263. J.M. acknowledges partial support from an Army Research Office MURI grant on AbInitio Solid-State Quantum Materials: Design, Production and Characterization at the Atomic Scale. The ADF-STEM imaging with TEAM 0.5 was performed at the Molecular Foundry, which is supported by the Office of Science, Office of Basic Energy Sciences of the U.S. DOE under Contract No. DE-AC0205CH11231. 
325 Author contributions J.M. directed the project; Yonggang Yao and L.H. synthesized the samples; J.Z., 326 P.E., A.K.S. and J.M. discussed and/or carried out the experiments; M.P., Yakun Yuan, A.R., S.J.O. and 327 J.M. developed the RESIRE algorithm. Yao Yang, F.Z., Yakun Yuan, D.J.C., J.Z., D.S.K., X.T. and J.M. 328 performed image reconstruction, atom tracing and classification, analysed the data and/or interpreted the 329 results; J.M., Yao Yang, J.Z., and F.Z. wrote the manuscript. All authors commented on the manuscript.

330 Competing interests The authors declare no competing interests.

\section{Addition information}

332 Supplementary Information (with a link to the experimental data, RESIRE reconstruction and data

333 analysis source codes: https://github.com/AET-MetallicGlass/Supplementary-Data-Codes) accompanies 334 the paper.

335 Correspondence and requests for materials should be addressed to J.M. (miao@physics.ucla.edu).

336 Data availability The experimental data set as well as the Matlab source codes for the RESIRE 337 reconstruction and data analysis described in Methods are accompanied with this submission as 338 supplementary information. The 3D atomic coordinates of the metallic glass nanoparticle will be deposited 339 in the Materials Data Bank (http://www.materialsdatabank.org) immediately after this work is published.

$340 \quad$ Figures and Figure legends

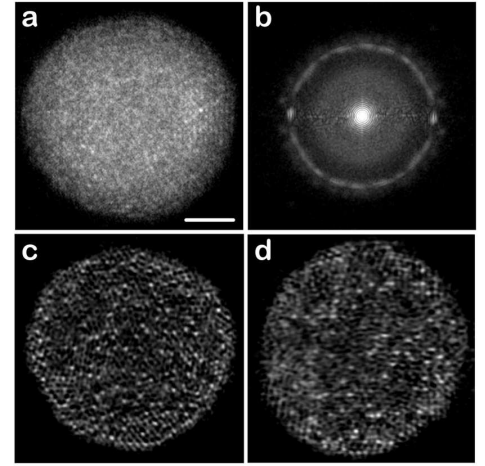

g

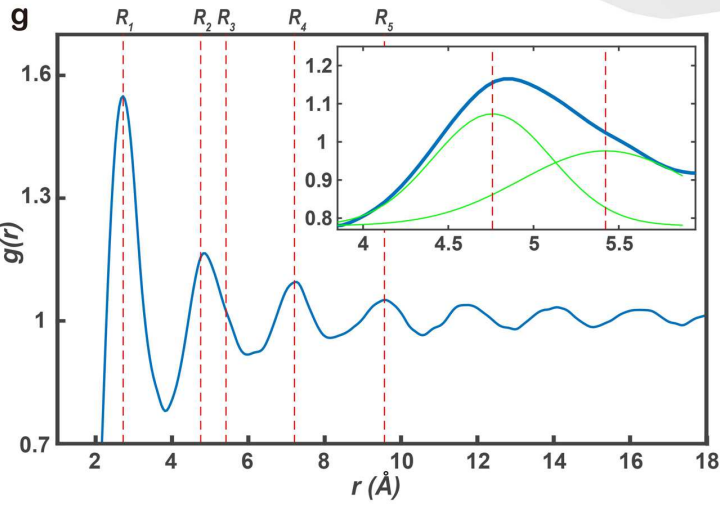

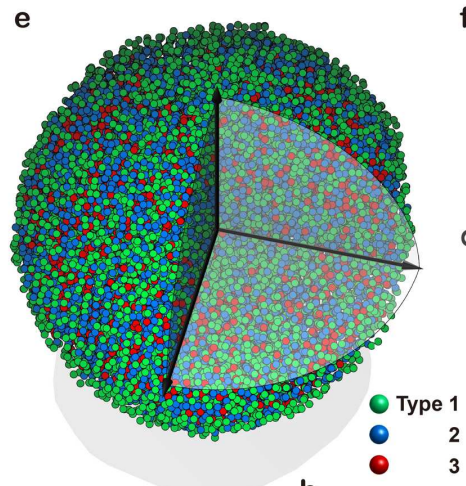

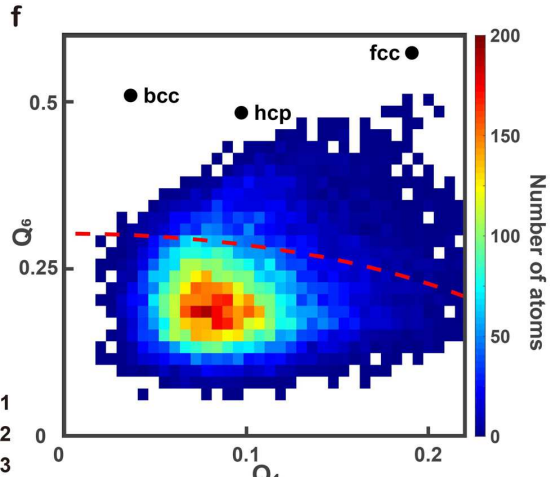

h

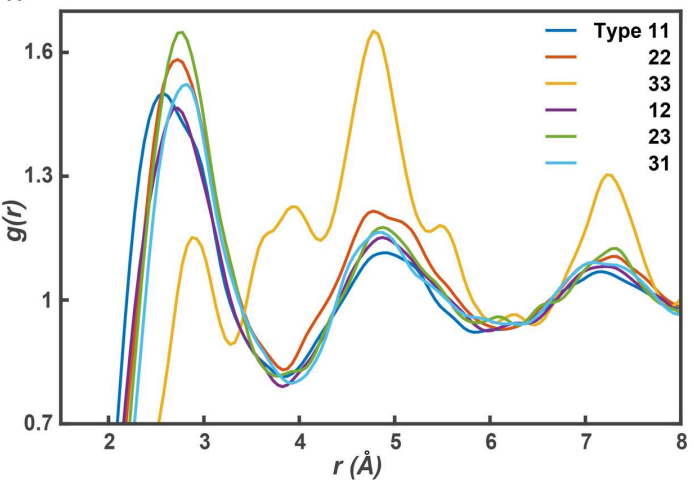


342 Figure 1 | Determining the 3D atomic structure of a multi-component metallic glass

343 with AET. a, A representative experimental image, where some crystalline features are

344 visible. b, Average diffraction pattern obtained from 55 experimental images (Extended

345 Data Fig. 3a), showing the amorphous halo. c, d, Two 2.4- $\AA$-thick slices of the 3D

346 reconstruction in the $x y$ - and $y z$-plane, respectively, where the majority of type 3 atoms

347 (bright dots) are distributed in the second coordination shell. e, Experimental 3D atomic

348 model of the metallic glass nanoparticle. f, The local BOO parameters of all the atoms in

349 the nanoparticle. Based on the criterion of the normalized BOO parameter $<0.5$ (the

350 dashed red curve), $84.54 \%$ of the total atoms are disordered. $\mathbf{g}$, The RDF of the disordered

351 atoms with the first, second, third, fourth and fifth peak positions $\left(R_{1}, R_{2}, R_{3}, R_{4}\right.$ and $\left.R_{5}\right)$

352 at $2.73,4.76,5.42,7.22$ and $9.57 \AA$, respectively, which are in good agreement with

353 those in the RDF directly derived from the average diffraction pattern (Extended Data

354 Fig. 3c). The inset shows the second-peak splitting with a Gaussian fit. h, The partial

355 PDFs between type 1, 2 and 3 atoms, consisting of 6 pairs - type 11, 12, 13, 22, 23 and

35633 atoms. The partial PDF for the type 33 atoms (the yellow curve) shows a unique feature

357 with a higher second peak than the first peak. 

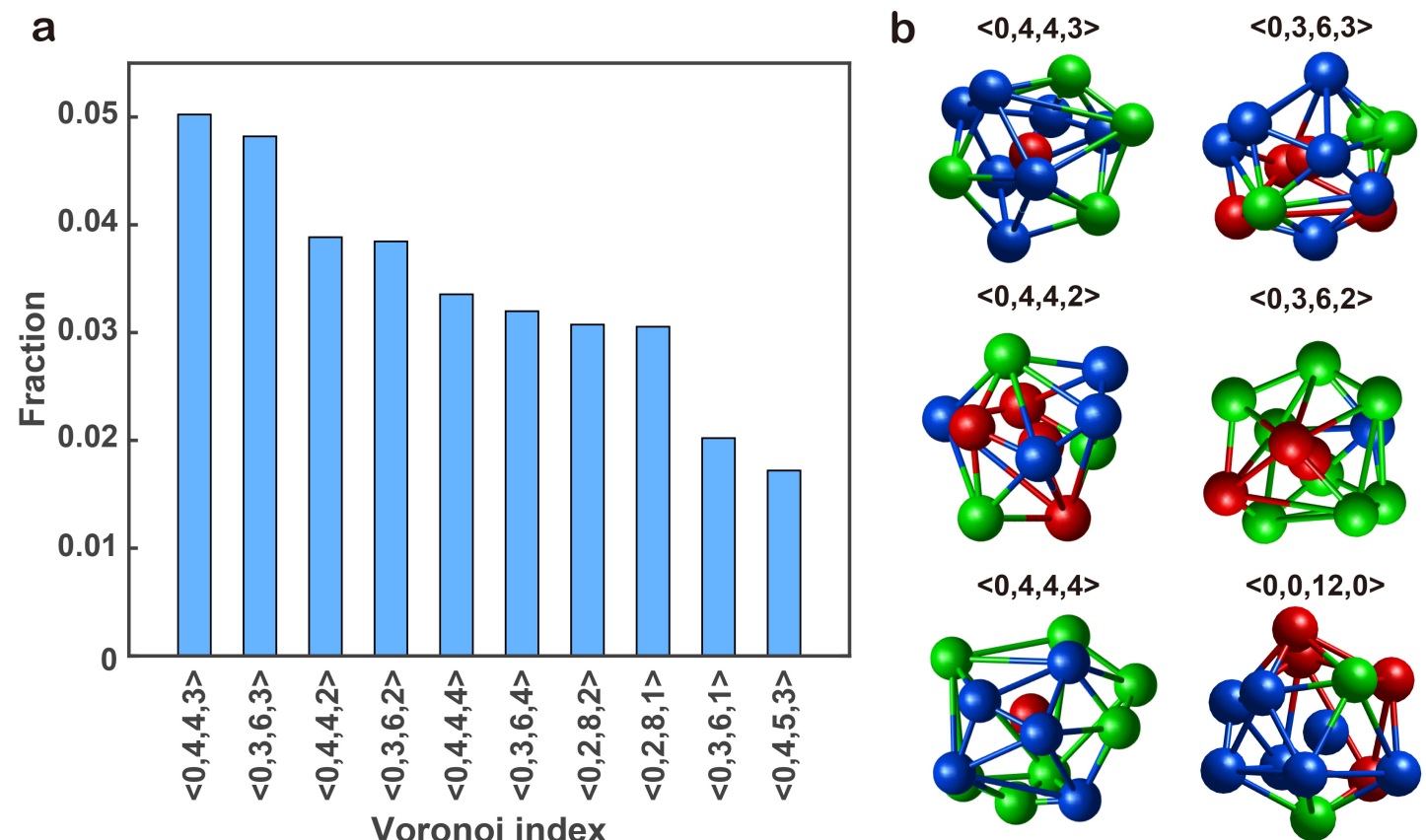

$<0,3,6,2>$
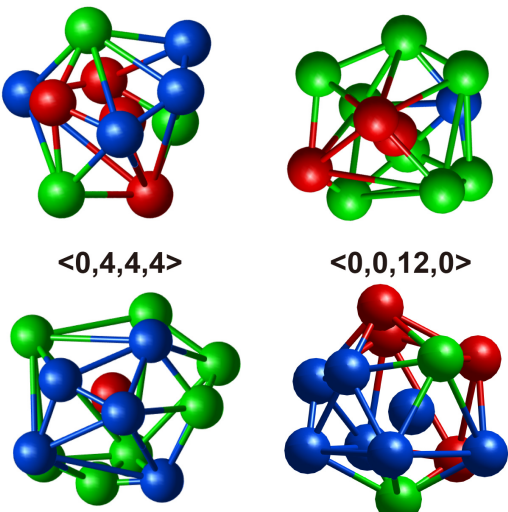

$<0,0,12,0\rangle$
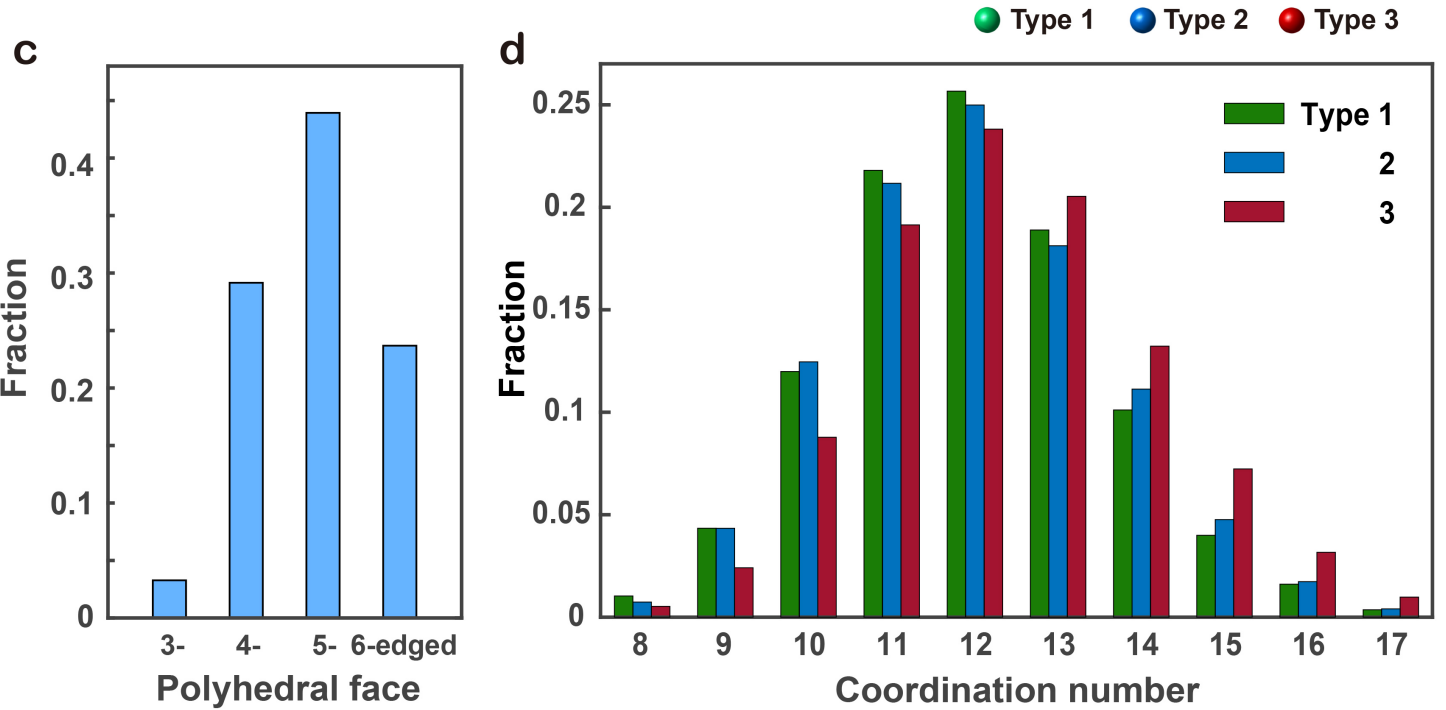

Polyhedral face

359 Figure 2 | The short-range order of the metallic glass nanoparticle. a, Ten most

360 abundant Voronoi polyhedra in the nanoparticle. b, Six representative Voronoi polyhedra,

361 where $<0,4,4,3>,<0,3,6,3>,<0,4,4,2>$ and $<0,3,6,2>$ are the four highest fraction Voronoi

362 indices, $<0,4,4,4>$ shows a severely distorted polyhedron, and $<0,0,12,0>$ represents an

363 icosahedron. c, The 3-, 4-, 5- and 6-edged face distribution of all the Voronoi polyhedra,

364 where the 5-edged faces are the most abundant (43.91\%). d, The coordination number

365 (CN) distribution for type 1, 2 and 3 atoms. 

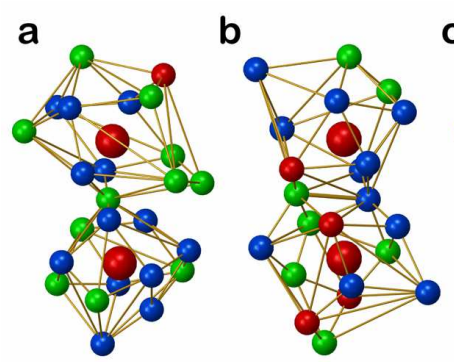

g

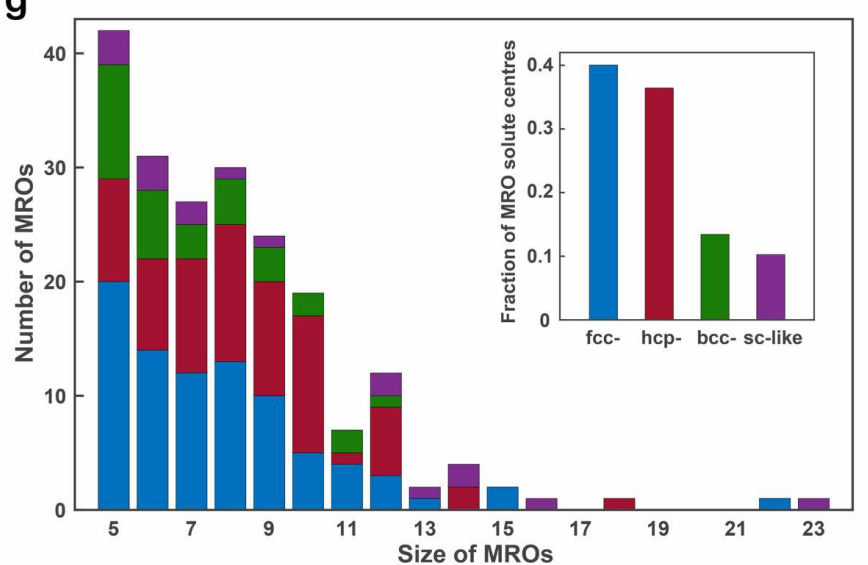

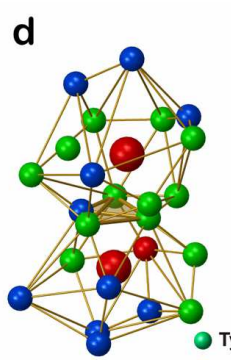

Type 1
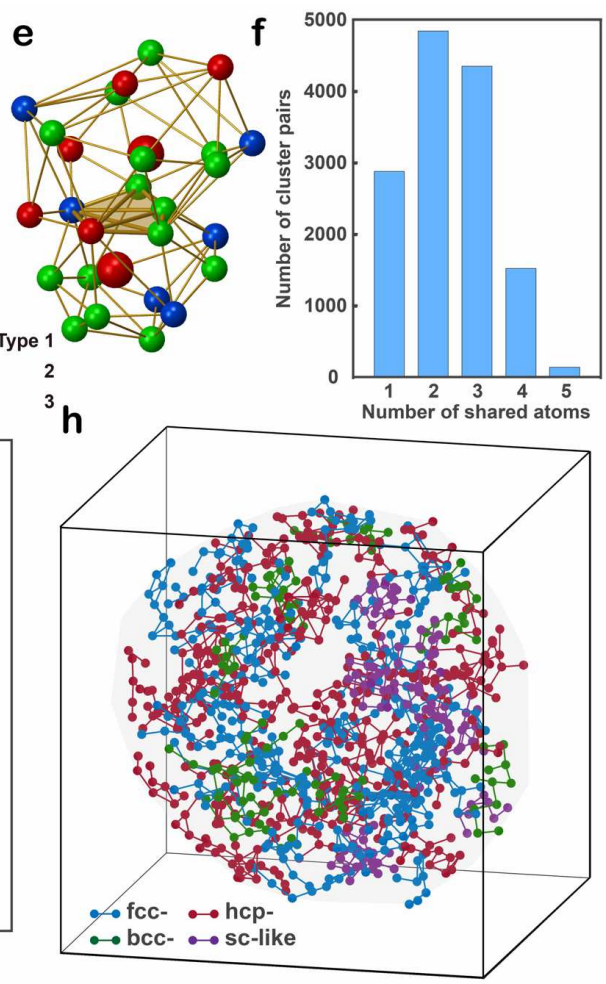

Figure 3 | The connectivity and distribution of the MRO in the metallic glass

nanoparticle. a-e, Representative pairs of the solute-centred clusters that are connected

with each other by sharing one, two, three, four and five atoms, respectively, where the

central atom of each cluster is indicated by a large red sphere. $\mathbf{f}$, Statistical distribution of the number of the solute-centred cluster pairs, which share from one to five atoms. $\mathbf{g}$, Histogram of the four types of MRO - fcc- (in blue), hcp- (in red), bcc- (in green) and sclike (in purple) - as a function of the size (i.e. the number of solute centres). The total number of fcc-, hcp-, bcc- and sc-like MRO is 85, 71, 31 and 17, respectively. The inset

375 shows the fraction of the solute centre atoms in the four types of MRO. $\mathbf{h}$, Distribution of the four types of the MRO with eight solute centre atoms or more, where the centre region lacks of large MRO. 

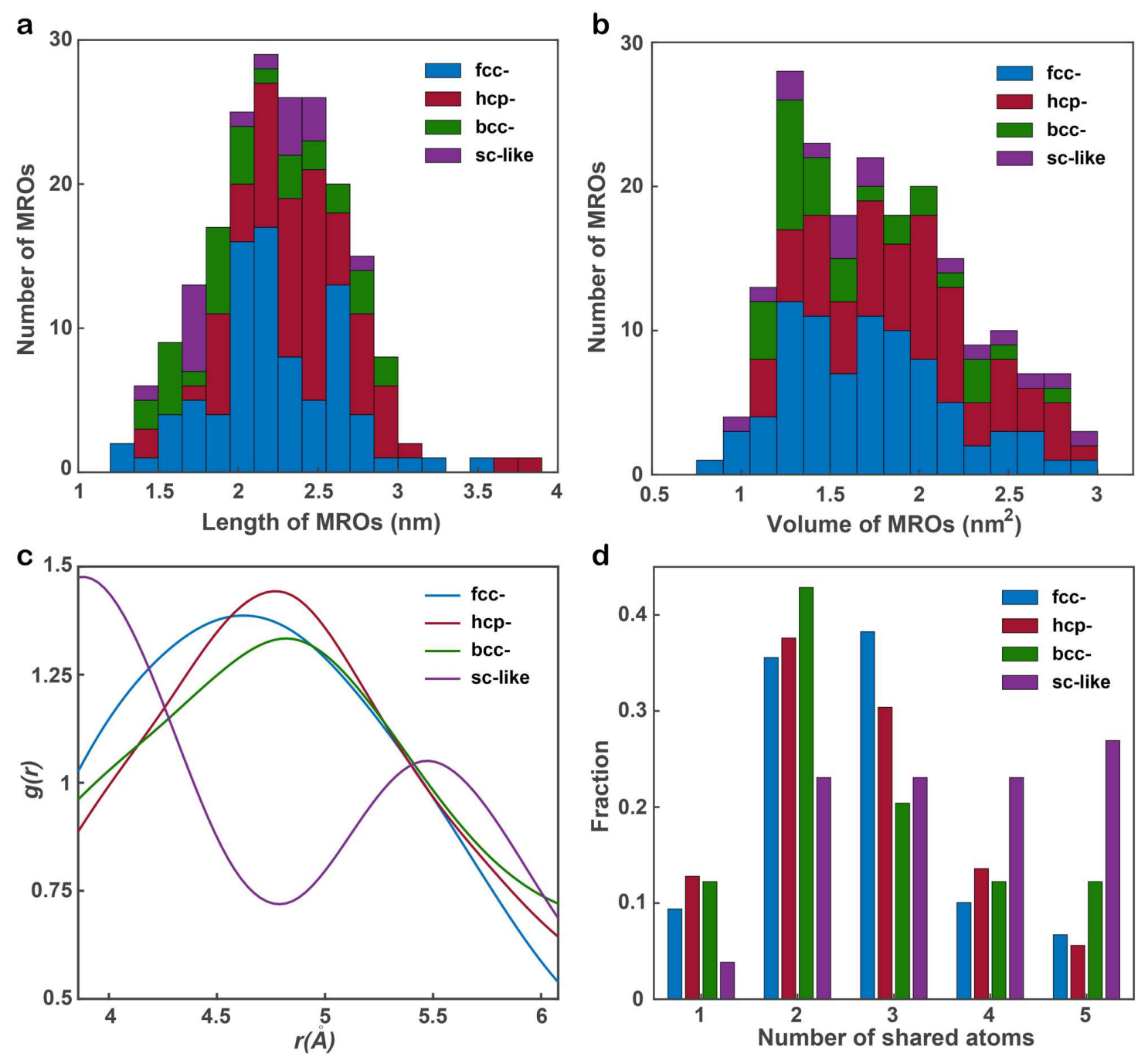

379 Figure 4 | Quantitative characterization of the MRO. The length (a) and volume (b)

380 distribution of the four types of the MRO in the metallic glass nanoparticle, where the

381 length was measured along the longest direction of each MRO. c, Partial PDFs of the fcc-

382 , hcp-, bcc- and sc-like solute centres in the metallic glass nanoparticle, where the

383 maximum peak positions are located at 4.62, 4.77, 4.82 and $3.88 \AA$, respectively.

384 Compared with the other three partial PDFs, the partial PDF of the sc-like solute centres

385 (the purple curve) shows two peaks with the ratio of the second to the first peak position

386 about $\sqrt{2}$. d, Distribution of sharing one, two, three, four and five atoms between

387 neighbouring solute-centred clusters for the four types of the MRO. 


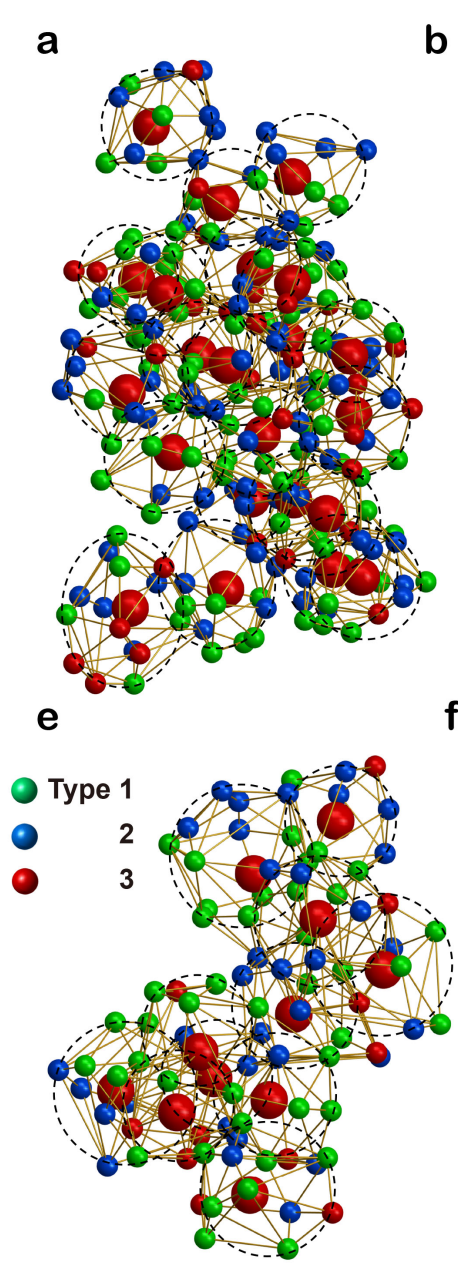

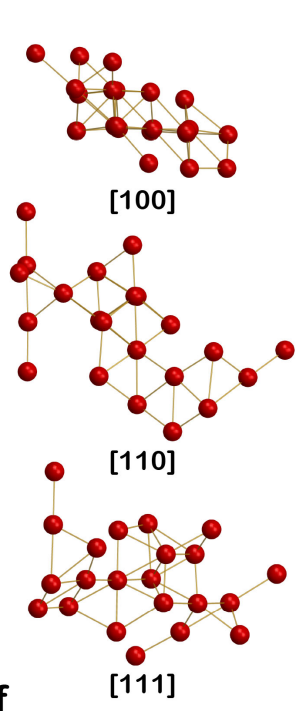
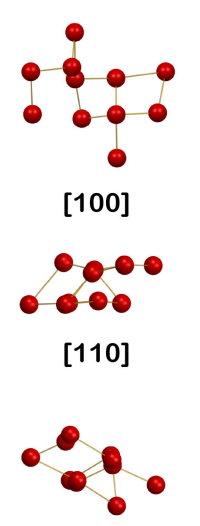

[111]
C
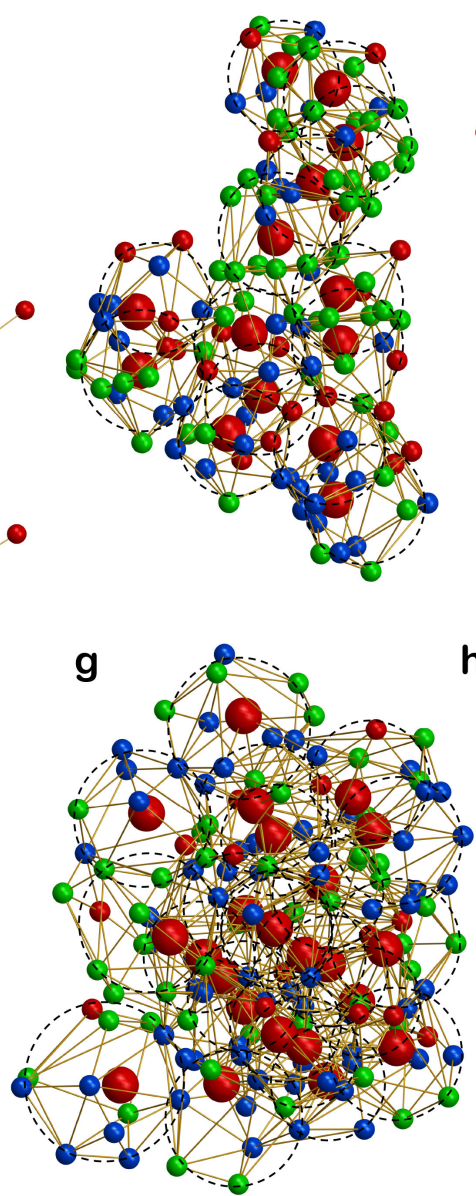

388

Figure 5 | 3D atomic packing of four representative MRO. Representative fcc- (a), hcp- (c), bcc- (e) and sc-like (g) MRO, consisting of 22, 14, 11 and 23 solute centres (large red spheres), respectively, where the solute-centred clusters (dashed circles) show only translational but no orientational order. To better visualize the crystal-like MRO, the solvent atoms are removed and the solute centres are orientated along the fcc (b), hcp (d), bcc (f) and sc (h) zone axes, showing that the 3D shapes of the MRO are anisotropic and the networks significantly deviate from perfect crystal lattices.

\section{METHODS}

Sample preparation. The multi-component metallic nanoparticle samples were synthesized using the thermal shock procedures published elsewhere ${ }^{43}$. Individual metal salts (chlorides or their hydrate forms) 
399 were dissolved in ethanol at a concentration of $0.05 \mathrm{~mol} / \mathrm{L}$. After complete dissolving with hydrochloric 400 acid, the individual salt precursor solutions with different cations were mixed and sonicated for 30 minutes.

401 The homogenously mixed precursor solution was loaded onto the carbon substrates ${ }^{51}$ (reduced graphene 402 oxide) and heated to a temperature as high as 1,763 K for 55 milliseconds (Extended Data Fig. 1a). The 403 sample was suspended on a trench and connected with copper electrodes by silver paste for both heating 404 and effective cooling as a giant heat sink. The thermal shock synthesis was triggered by electric Joule 405 heating in an argon-filled glovebox using a Keithley 2425 SourceMeter where the high temperature and 406 duration can be effectively controlled by tuning the input power and duration. The temperature of this 407 process was monitored by a high-speed Phantom Miro M110 camera with a pixel size of $25 \mu \mathrm{m}$ 408 (Supplementary Video 1). The cooling rate was estimated to be $\sim 5.1-6.9 \times 10^{4} \mathrm{~K} / \mathrm{s}$ (Extended Data Fig. 1a), 409 which, according to previous studies, can make metallic glasses ${ }^{52,53}$. The resulting nanoparticles on reduced 410 graphene oxide were dispersed in ethanol with sonication. After deposited on to 5-nm-thick silicon nitride 411 membranes, the nanoparticles were baked at $100{ }^{\circ} \mathrm{C}$ for 12 hours in vacuum to eliminate any hydrocarbon 412 contamination. Both energy-dispersive X-ray and electron energy loss spectroscopy data show that the 413 nanoparticles were still in metallic form and were not oxidized during the experiment (Extended Data Fig. 414 1b-q).

415 Data acquisition. A set of tomographic tilt series were acquired from seven nanoparticles using the TEAM 4160.5 microscope with the TEAM stage ${ }^{54}$. Images were collected at $200 \mathrm{kV}$ in ADF-STEM mode (Extended 417 Data Table 1). To minimize sample drift, four sequential images per tilt angle were measured with a dwell 418 time of $3 \mu \mathrm{s}$. To monitor any potential damage induced by the electron beam, we took $0^{\circ}$ images before, 419 during and after the acquisition of each tilt series and ensured that no noticeable structural change was 420 observed for the seven nanoparticles. The total electron dose of each tilt series was estimated to be between $4217 \times 10^{5} \mathrm{e}^{-/} \AA^{2}$ and $9.5 \times 10^{5} \mathrm{e}^{-/} \AA^{2}$ (Extended Data Table 1).

422 Image pre-processing and denoising. For each experimental tilt series, we performed the following 423 procedure for image post-processing and denoising.

424 i) Image registration. At each tilt angle, we used the first image as a reference and calculated normalized 425 cross-correlation between the reference and the other three images using a step size of 0.1 pixel $^{55}$. These 426 four images were aligned and summed to form an experimental image at that tilt angle.

427 ii) Scan distortion correction ${ }^{38}$. Two steps were used to correct the scan distortion for the experimental 428 images. First, a set of low-magnification images were taken from nanoparticles and their positions were 429 fitted with a Gaussian. Based on the geometric relation of the nanoparticles at different angles, the scan coil 430 directions were calibrated to be perpendicular and equal in strength. Second, six high-magnification images 431 were taken from a multi-component metallic nanoparticle and scan distortion parameters were estimated 432 by minimizing the mean squared error of the common line of the six images. These scan distortion 433 parameters were applied to the experimental images.

434 iii) Image denoising. The experimental images contain mixed Poisson and Gaussian noise and were 435 denoised by the block-matching and 3D filtering (BM3D) algorithm ${ }^{56}$, which has been demonstrated to be 436 effective in reducing noise in $\mathrm{AET}^{38,40,42}$. The BM3D denoising parameters were optimized by the following 437 three steps. First, Poisson and Gaussian noise level were estimated from the experimental tilt series. Second, 
438 several images were simulated based on a model nanoparticle, which has a similar size and elemental

439 distribution as those of an experimental image. The same level of Poisson and Gaussian noise was added

440 to the simulated images. Third, these noisy images were denoised by BM3D with different parameters. The

441 denoising parameters corresponding to the largest cross-correlation coefficient between the denoised and

442 the original images were chosen and applied to denoise the experimental images.

443 iv) Background subtraction and alignment. After denoising, a 2D mask was defined from each experimental

444 image, which is slightly larger than the size of the nanoparticle. The background inside the mask was

445 estimated by the discrete Laplacian in Matlab. After background subtraction, the experimental images of

446 each tilt series were projected onto the tilt axis to produce a set of 1D curves (termed common lines). The

447 images were aligned along the tilt axis by maximizing the cross-correlation between the common lines. The

448 alignment of the images perpendicular to the tilt axis was achieved by the centre of mass method. The

449 centres of mass of the images were calculated and the images were shifted so that all the centres of mass

450 coincide with the origin. This image alignment method has been successfully used to achieve sub-pixel

451 accuracy $y^{34,36,40-42}$. The Matlab data of the raw, processed and aligned experimental images are provided in

452 Supplementary Information.

453 The REal Space Iterative REconstruction (RESIRE) algorithm. After post-processing and denoising,

454 the experimental images were reconstructed by the RESIRE algorithm. The algorithm iteratively minimizes

455 an error function defined by,

$$
\varepsilon_{\theta}(O)=\frac{1}{2} \sum_{x, y}\left|\Pi_{\theta}(O)\{x, y\}-b_{\theta}\{x, y\}\right|^{2}
$$

where $\varepsilon_{\theta}(O)$ is an error function of a $3 \mathrm{D}$ object $(O)$ at tilt angle $\theta, \Pi_{\theta}(O)$ projects $O$ to generate a $2 \mathrm{D}$ image at angle $\theta, b_{\theta}$ is the experimental image at angle $\theta$, and $\{x, y\}$ is the coordinates. The minimization is solved via the gradient descent,

$$
\nabla \varepsilon_{\theta}(O)\{u, v, w\}=\Pi_{\theta}(O)\{x, y\}-b_{\theta}\{x, y\} \text { where }\left[\begin{array}{c}
u \\
v \\
w
\end{array}\right]=R_{\theta}\left[\begin{array}{l}
x \\
y \\
z
\end{array}\right] \text { for some } z
$$

where $\nabla$ represents the gradient and $R_{\theta}$ is the rotation matrix at tilt angle $\theta$, which transforms coordinates $\{x, y, z\}$ to $\{u, v, w\}$. The $\mathrm{j}^{\text {th }}$ iteration of the RESIRE algorithm consists of the following four steps.

i) A set of images are calculated from the $3 \mathrm{D}$ object of the $\mathrm{j}^{\text {th }}$ iteration using a Fourier method. The $3 \mathrm{D}$ object is first padded with zeros by properly choosing an oversampling ratio ${ }^{57}$. Applying the fast Fourier transform to the zero-padded object generates a 3D array in reciprocal space, from which a series of 2D Fourier slices are obtained at different tilt angles. These 2D Fourier slices are inverted to a set of images

467 via the inverse Fourier transform.

468 ii) The error function defined in equation 1 is calculated between the computed and experimental images.

469 iii) The gradient of the error function is computed for every voxel using equation 2.

470 iv) The $3 \mathrm{D}$ object of the $(\mathrm{j}+1)^{\text {th }}$ iteration is updated by,

$$
O^{j+1}=O^{j}-\frac{\Delta}{n N} \sum_{\theta} \nabla \varepsilon_{\theta}\left(O^{j}\right)
$$


472 where $\Delta$ is the step size ( $\Delta=2$ was chosen for the reconstruction of our experimental data), $n$ is the number 473 of images and $N$ is the dimension of each image $(N \times N) . O^{j+1}\{u, v, w\}$ is used as an input for the $(\mathrm{j}+1)^{\mathrm{th}}$ 474 iteration.

475 The convergence of the algorithm is monitored by the R-factor,

$$
R=\frac{1}{n} \sum_{\theta} \frac{\sum_{x, y}\left|\Pi_{\theta}(O)\{x, y\}-b_{\theta}\{x, y\}\right|}{\sum_{x, y}\left|b_{\theta}\{x, y\}\right|} .
$$

Usually, after several hundreds of iterations, the algorithm converges to a high-quality 3D reconstruction from a limited number of images. Both our numerical simulation and experimental results have indicated that RESIRE outperforms other iterative tomographic algorithms such as generalized Fourier iterative reconstruction $^{58}$ and simultaneous iterative reconstruction technique ${ }^{59}$. By avoiding iterating between real and reciprocal space, RESIRE can be applied to general sample geometry such as thin films and extended objects. The details of the RESIRE algorithm will be reported in a follow-up paper.

For each aligned experimental tilt series, we first ran RESIRE for 200 iterations. From the initial 3D reconstruction, we performed the angular refinement and spatial alignment for the experimental images $^{40,58}$. For each experimental image, we determined the corresponding three Euler angles of the 3D reconstruction. We sequentially scanned each of the three Euler angles with a small angular increment. At each scanning step, we projected the 3D reconstruction back to calculate an image. The experimental image was shifted along the $\mathrm{x}$ and $\mathrm{y}$-axis and aligned with the calculated one. An error metric, defined as the difference between the calculated and experimental image, was computed. After scanning all the three Euler angles, three optimal Euler angles was found with the smallest error metric. This procedure was iterated for all the experimental images until there was not further improvement, producing a set of spatially aligned experimental images and refined tilt angles. Next, the background of each experimental image was reevaluated and re-subtracted. Using these experimental images with the refined tilt angles (Extended Data Fig. 4a), we ran another 200 iterations of RESIRE to obtain the final 3D reconstruction of each experimental tilt series (Extended Data Table 1). The source codes of RESIRE are provided in Supplementary Information.

Determination of 3D atomic coordinates and species. From each final 3D reconstruction, the atomic coordinates and species were identified using the following procedure ${ }^{40,42}$.

i) Each 3D reconstruction was upsampled by a factor of 3 using the spline interpolation, from which all the local maxima were identified. Starting from the highest intensity peak, polynomial fitting ${ }^{60}$ was performed on a $0.8 \times 0.8 \times 0.8 \AA^{3}(7 \times 7 \times 7$ voxel $)$ volume around each local maximum to locate the peak position. If the distance between the fitted peak position and existing potential atom positions is larger than or equal to 2 $\AA$, it was listed as a potential atom. After repeating this step for all the local maxima, a list of potential atom positions was obtained. This method to trace the positions of potential atoms has previously been rigorously tested by using two independent experimental tilt series acquired from the same sample ${ }^{42}$. of the potential atoms. Based on the difference map, we manually adjusted a very small fraction of the atoms (167 out of 18356), which has been routinely used in protein crystallography ${ }^{61}$. 
509 iii) A K-mean clustering method ${ }^{40,42,62}$ was used to classify three types of atoms and non-atoms (Co and Ni 510 as type 1, Ru, Rh, Pd and Ag as type 2, and Ir and Pt as type 3) based on the integrated intensity of a $0.8 \AA$ $511 \times 0.8 \AA \times 0.8 \AA$ volume around each potential atom position. An initial atomic model with $3 \mathrm{D}$ atomic 512 coordinates was determined from each 3D reconstruction.

513 iv) Due to the missing wedge problem and noise in the experimental images, there is local intensity 514 variation in each 3D reconstruction. A local reclassification was iteratively performed to refine the type 1,

5152 and 3 atoms. Each atom was defined as the centre of a 10-A-radius sphere. The average intensity 516 distribution of type 1, 2 and 3 atoms was computed within the sphere. The $\mathrm{L}_{2}$ norm of the intensity 517 distribution between the centre atom and the average type 1,2 and 3 atom was calculated. The centre atom 518 was assigned to the type with the smallest $\mathrm{L}_{2}$ norm. The procedure was iteratively repeated until there were 519 no further changes. The source codes for 3D atom tracing and classification are provided in Supplementary 520 Information.

521 Refinement of 3D atomic coordinates. The 3D atomic coordinates were refined by minimizing the error 522 between the calculated and measured images using the gradient descent ${ }^{38,40,42}$. Each atom was first fit with 523 a 3D Gaussian function with a height $H$ and a width $B^{\prime}$, where $H$ and $B^{\prime}$ were considered the same for the 524 same type of atoms. A 3D atomic model was obtained by,

$$
O\{x, y, z\}=\sum_{i} H_{i} \exp \left[-\frac{\left|x-x_{i}\right|^{2}+\left|y-y_{i}\right|^{2}+\left|z-z_{i}\right|^{2}}{B_{i}^{\prime}}\right]
$$

where $x_{i}, y_{i}, z_{i}, H_{i}$ and $B_{i}^{\prime}$ are the coordinates, height and standard deviation of the $i^{\text {th }}$ atom, respectively, $\left|x-x_{i}\right|,\left|y-y_{i}\right|,\left|z-z_{i}\right| \leq \rho$, and $\rho$ is a cut-off size of the 3D Gaussian function. From the 3D atomic model, a set of projection images were computed at different tilt angle $\theta$ by,

$$
\Pi_{\theta}(O)\{u, v\}=\sum_{w} \sum_{i} H_{i} \exp \left[-\frac{\left|u-u_{i}\right|^{2}+\left|v-v_{i}\right|^{2}+\left|w-w_{i}\right|^{2}}{B_{i}^{\prime}}\right]
$$

$$
\text { where }\left[\begin{array}{l}
u_{i} \\
v_{i} \\
w_{i}
\end{array}\right]=R_{\theta}\left[\begin{array}{c}
x_{i} \\
y_{i} \\
z_{i}
\end{array}\right] \text { and }\left|u-u_{i}\right|,\left|v-v_{i}\right|,\left|w-w_{i}\right| \leq \rho \text {. }
$$

Substituting equation (6) into (1), an error function was calculated, from which the gradient descent method was used to search for the optimal atomic position at the $(j+1)^{\text {th }}$ iteration,

$$
\left\{x_{i}, y_{i}, z_{i}\right\}^{j+1}=\left\{x_{i}, y_{i}, z_{i}\right\}^{j}-\Delta \sum_{\theta}\left[\Pi_{\theta}(O)\{u, v\}-b_{\theta}\{u, v\}\right] \nabla_{i}\left[\Pi_{\theta}(O)\{u, v\}\right]
$$

534 Where $\nabla_{i}$ is the spatial gradient operator with respect to the atomic position $\left(x_{i}, y_{i}, z_{i}\right)$. The iterative refinement process was terminated when the $\mathrm{L}_{2}$ norm error could not be further reduced.

536 The local bond orientational order (BOO) parameters. The local BOO parameters $\left(\mathrm{Q}_{4}\right.$ and $\left.\mathrm{Q}_{6}\right)$ were 537 calculated from the 3D atomic model of each nanoparticle using a method described elsewhere ${ }^{63,64}$. The $\mathrm{Q}_{4}$ and Q6 order parameters were computed up to the second shell with a shell radius set by the first valley in the RDF curve of the 3D atomic model. Figure $1 \mathrm{f}$ and Extended Data Fig. 2h-n show the distribution of the local BOO parameters of all the atoms in particles 1-7. To separate the amorphous structure from the crystal nuclei, we calculated the normalized local BOO parameter, defined as $\sqrt{\mathrm{Q}_{4}{ }^{2}+\mathrm{Q}_{6}{ }^{2}} / \sqrt{\mathrm{Q}_{4 \mathrm{fcc}}{ }^{2}+\mathrm{Q}_{6 \mathrm{fcc}}{ }^{2}}$, where $Q_{4 \text { fcc }}$ and $Q_{6 \text { fcc }}$ are the $\mathrm{Q}_{4}$ and $\mathrm{Q}_{6}$ value for a perfect fcc lattice. The normalized BOO parameter is 
543 between 0 and 1 , where 0 means $\mathrm{Q}_{4}=\mathrm{Q}_{6}=0$ and 1 represents a perfect fcc crystal structure. Based on the

544 BOO parameters of a $\mathrm{Cu}_{65} \mathrm{Zr}_{35}$ metallic glass structure obtained from molecular dynamics simulations ${ }^{65}$

545 (Extended Data Fig. 20), we chose the normalized BOO parameter $=0.5$ as a cut-off to separate crystal

546 nuclei from amorphous structure (red curves in Fig. 1f and Extended Data Fig. 2h-n).

547 3D precision estimation with multislice simulations. A tilt series of 55 STEM images were calculated

548 from the experimental 3D atomic model by using a fast multislice simulation software based on graphics

549 processing unit ${ }^{66}$. At each refined experimental angle (Extended Data Fig. 4a), the experimental 3D atomic

550 model was placed in a cuboidal super cell and the super cell was divided into multiple 2- $\AA$-thick slices

551 along the z-axis. The experiment parameters shown in Extended Data Table 1 (particle 1) were used for the

552 multislice simulations. After using the parallel computing to perform the multislice simulations for all the

553 angles, we calculated 55 multislice STEM images, each with $289 \times 289$ pixels and a pixel size of $0.347 \AA$.

554 To account for the electron probe size and other incoherent effects, each multislice STEM image was

555 convolved with a Gaussian kernel. Extended Data Fig. 4c and d show a representative experimental and

556 multislice STEM image, respectively. An average R-factor between the 55 experimental and multislice

557 images (defined in equation 4) was computed to be $14.96 \%$, which, according to the crystallography

558 standard $^{61}$, represents a good agreement between the two sets of images.

559 From the 55 multislice STEM images with angular errors (Extended Data Fig. 4a), we performed

560 the 3D reconstruction and angular refinement with RESIRE (Extended Data Fig. 4b). After applying the

561 atomic tracing, classification and refinement procedure to the reconstruction, we obtained a new 3D atomic

562 model of the sample, consisting of 8438, 6905 and 3138 type 1, 2, and 3 atoms, respectively. We identified

$5637898,6837,3138$ common pairs of type 1, 2 and 3 atoms, respectively, between the experimental and

564 multislice atomic models based on the criterion of each common pair within a radius of $1.5 \AA$. The total

565 common pairs of the three types of atoms are 17873 , indicating that $97.37 \%$ of all atoms have been corrected

566 identified. Extended Data Fig. 4d shows the distribution of the atomic deviation between all the common

567 pairs with a root-mean-square deviation (i.e. 3D precision) of $21 \mathrm{pm}$.

568 The radial distribution function (RDF) and partial pair distribution function (PDF). The RDF was

569 calculated for the 3D atomic model of each nanoparticle using the following procedure. i) The distance of

570 all atom pairs in each 3D atomic model was computed and binned into a histogram. ii) The number of atom

571 pairs in each bin was normalized with respect to the volume of the spherical shell corresponding to each

572 bin. iii) The histogram was scaled so that the RDF approaches one for large separations. After plotting the

573 RDF for each nanoparticle, the first valley of the RDF was used as the nearest neighbour cut-off distance

574 to calculate the local BOO parameters (Fig. 1f and Extended Data Fig. 2h-n). By choosing the atoms in the

575 metallic glass nanoparticle (particle 1) with the normalized BOO parameter $<0.5$, we applied the above

576 procedure to plot the RDF (Fig. 1g). For type 1, 2 and 3 atoms, we identified six sets of atoms pairs (type

$57711,12,13,22,23$ and 33) in the nanoparticle. For each set of atom pairs, we used the above procedure to

578 calculate the partial PDF shown in Fig. 1h.

579 Voronoi tessellation and the coordination number $(\mathbf{C N})$. The analysis of Voronoi tessellation was

580 performed by following the procedure published elsewhere ${ }^{6}$, where the surface atoms of the nanoparticle

581 were excluded. To reduce the effect of the experimental and reconstruction error on Voronoi tessellation, 
582 those surfaces with areas less than $1 \%$ of the total surface area of each Voronoi polyhedron were removed ${ }^{9}$.

583 From the Voronoi tessellation, each polyhedron is designated by a Voronoi index $\left\langle n_{1}, n_{2}, n_{3}, n_{4}, \cdots\right\rangle$ with

$584 n_{i}$ denoting the number of $i$-edged faces and the $\mathrm{CN}$ was calculated by $\sum_{i} n_{i}$.

585 Quantification of the chemical SRO. We used the Warren-Cowley parameters $\left(\alpha_{l m}\right)$ to quantify the 586 chemical $\mathrm{SRO}^{67,68}$,

$$
\alpha_{l m}=1-\frac{Z_{l m}}{\chi_{m} Z_{l}}
$$

588 where $l, m=1,2$ or $3, Z_{l m}$ is the partial $\mathrm{CN}$ of type $m$ atoms around type $l$ atoms, $\chi_{m}$ is the fraction of 589 type $m$ atoms, and $Z_{l}$ is the total $\mathrm{CN}$ around type $l$ atoms. After excluding the surface atoms, we estimated $590 \quad \chi_{1}, \chi_{2}$ and $\chi_{3}$ to be $42.97 \%, 38.28 \%$ and $18.75 \%$, respectively. Using the partial CNs (Extended Data Fig. 5915 b), we calculated $\alpha_{11}=-0.11, \alpha_{12}=0.1, \alpha_{13}=0.05, \alpha_{21}=0.02, \alpha_{22}=0.01, \alpha_{23}=-0.07, \alpha_{31}=0.03, \alpha_{32}$ $592=-0.06$, and $\alpha_{33}=0.06$, indicating that the type 11 and 23 bonds are favoured, but the type 12 and 33 bonds are unfavoured. These results are consistent with the observations that the type 23 bond is $0.06 \AA$ shorter than the average type 2 and 3 bonds and the type 12 bond is $0.06 \AA$ longer than the average type 1 and 2 bonds (Fig. 1h).

596 Determination of the solute centres and the MRO. A breadth-first-search algorithm ${ }^{69,70}$ was implemented 597 to search for the solute centres and the MRO using the following procedure. First, the algorithm identified the solute centres from type 3 atoms based on two criteria: i) each solute centre must fall within a $0.75 \AA$ radius from an fcc, hcp, bcc or sc lattice point; and ii) each solute centre must have at least one neighbouring type 3 atom within the second-coordination-shell distance. Second, the identified solute centres were sorted out to generate a queue of the fcc-, hcp-, bcc- or sc-like MRO candidates. Third, starting from the largest MRO candidate (i.e. with the most solute centres), each candidate was classified as an MRO if it has at least five or more solute centres and none of the solute centres was already occupied by another MRO. If any solute centres were already occupied, they were removed from the MRO candidate and the candidate was refitted into the lattice vectors and added back into the queue. If two or more MRO candidates have the same number of solute centres, the one with the smallest error of fitting the solute centres into the lattice vectors was analysed first. This process was repeated until all the MRO was identified, where each solute centre can only belong to no more than one MRO. To corroborate our analysis, we repeated the above steps with a $1 \AA$ and $0.5 \AA$ radius cut-off and the corresponding MRO is shown in Extended Data Figs. 6 and 7 , respectively.

611 An attempt was also made in searching for icosahedral-like MRO. The breadth-first-search algorithm ${ }^{69,70}$ was used to find the MRO that falls within a $0.75 \AA$ radius from the 12 vertices of an

613 icosahedron. Because the icosahedron cannot be periodically packed in three dimensions, only the nearest 614 neighbour vertices were searched, making the largest possible MRO have 13 solute centres ( 1 central solute 615 centre plus 12 nearest neighbours). After performing the search, the resulting possible MRO has a mean 616 value of 3.9, meaning on average each solute centre is connected to only 3 others when constrained to an 617 icosahedron within the second coordination shell. Furthermore, although the largest possible MRO has 7 618 solute centres, none of these solute centres form 5-fold symmetry. We also repeated this analysis with a 1 $619 \AA$ radius cut-off. The mean value of solute centres becomes 4.5, the largest MRO has 8 solute centres, and 
620 there are 195 -fold symmetries. The source codes for identifying the MRO are provided in Supplementary 621 Information.

622 Determination of the 3D atomic structure of an amorphous CuTa thin film. The following procedure 623 was used to experimentally resolve the $3 \mathrm{D}$ atomic positions in the $\mathrm{CuTa}$ thin film.

624 i) Sample preparation. CuTa thin films were fabricated in-situ in the sample chamber of the spin polarized 625 low energy electron microscope (SPLEEM) at NCEM, where clean ultrahigh vacuum conditions remained 626 in the low $10^{-9}$ torr range. Using thermal evaporation, CuTa thin films were deposited on $\mathrm{Si}_{3} \mathrm{~N}_{4} \mathrm{Substrates}_{\text {, }}$ 627 which were maintained well below $150 \mathrm{~K}$ during sample fabrication. The growth rate of the thin films was 628 in the range of $0.1-1$ atomic monolayer per minute. After the fabrication of the CuTa thin films, a very 629 thin carbon capping layer was deposited on the films to protect the samples from oxidation.

630 ii) Data acquisition. A tomographic tilt series was acquired from the CuTa thin film using TEAM I under 631 ADF-STEM mode at $300 \mathrm{kV}$. To mitigate the sample drift, two images at each tilt angle were taken and 632 then aligned to improve the signal-to-noise ratio. The tilt series consists of a total of 40 images with a tilt 633 range from $-67.9^{\circ}$ to $64.9^{\circ}$ (Extended Data Fig. 8). As the CuTa film is thinner than $\sim 6 \mathrm{~nm}, 40$ experimental 634 images are sufficient to produce a good $3 \mathrm{D}$ reconstruction. The total electron dose of the dataset is $4.8 \times 10^{5}$ $635 \mathrm{e} / \AA^{2}$. All the experimental parameters of the tilt series can be found in Extended Data Table 1.

636 iii) Image alignment. All the image pre-processing and denoising steps for the analysis of the CuTa thin 637 film are similar to those of the metallic glass nanoparticle, except for image alignment. We first used the 638 cross-correlation between the neighbouring images to roughly align the CuTa images. Next, we searched 639 for some reference markers, which can be either created by adding some small nanoparticles or based on 640 features in the sample. In this experiment, we chose an isolated region in the images and aligned them using 641 the centre of mass and common line method ${ }^{34,36}$. After obtaining the 3D reconstruction, we further refined 642 the alignment by projecting the reconstruction back to generate images and comparing them with the 643 experimental ones. This process was repeated until no further improvement could be made.

644 iv) 3D reconstruction, atomic tracing and refinement. Using RESIRE, we first performed a large volume 645 reconstruction of the $\mathrm{CuTa}$ thin film from the aligned images. Based on the thickness variation of the thin 646 film, we applied scanning $\mathrm{AET}^{41}$ to conduct multiple local volume reconstructions and then patched them 647 together to produce a full 3D reconstruction. Scanning AET has been previously demonstrated to be 648 effective in improving the $3 \mathrm{D}$ reconstruction of $2 \mathrm{D}$ materials and/or thin film samples ${ }^{41}$. From the full 3D 649 reconstruction, we projected it back to generate images and use them to perform the angular refinement and 650 spatial alignment. We iteratively repeated the process until there were no further changes. After obtaining 651 the final 3D reconstruction, we traced the $\mathrm{Cu}$ and $\mathrm{Ta}$ atoms based on the integrated intensity difference 652 between the two types of atoms. The 3D atomic positions were refined to produce a final 3D atomic model of the CuTa thin film (Extended Data Fig. 9).

654 51. Xu, Z., Sun, H., Zhao, X. \& Gao, C. Ultrastrong fibers assembled from giant graphene oxide sheets. Adv. Mater. 25, 188-193 (2013).

52. Takeuchi, A. \& Inoue, A. Quantitative evaluation of critical cooling rate for metallic glasses. Mater. Sci. Eng. A 304-306, 446-451 (2001). 
53. Bordeenithikasem, P. et al. Determination of critical cooling rates in metallic glass forming alloy libraries through laser spike annealing. Sci Rep 7, 7155 (2017).

54. Ercius, P., Boese, M., Duden, T. \& Dahmen, U. Operation of TEAM I in a user environment at NCEM. Microsc. Microanal. 18, 676-683 (2012).

55. Lewis, J. P. Fast normalized cross-correlation. Vis. Interface 95, 120-123 (1995).

56. Dabov, K., Foi, A., Katkovnik, V. \& Egiazarian, K. Image denoising by sparse 3-D transformdomain collaborative filtering. IEEE Trans. Image Process. 16, 2080-2095 (2007).

57. Miao, J., Sayre, D. \& Chapman, H. N. Phase retrieval from the magnitude of the Fourier transform of non-periodic objects, J. Opt. Soc. Am. A 15, 1662-1669 (1998).

58. Pryor, A. et al. GENFIRE: a generalized Fourier iterative reconstruction algorithm for highresolution 3D imaging. Sci. Rep. 7, 10409 (2017).

59. Gilbert, P. Iterative methods for the three-dimensional reconstruction of an object from projections. J. Theor. Biol. 36, 105-117 (1972).

60. Rogers, S. S., Waigh, T. A., Zhao, X. \& Lu, J. R. Precise particle tracking against a complicated background: polynomial fitting with Gaussian weight. Phys. Biol. 4, 220-227 (2007).

61. Brünger, A. T. et al. Crystallography \& NMR System: a new software suite for macromolecular structure determination. Acta Crystallogr. D54, 905-921 (1998).

62. Lloyd, S. Least squares quantization in PCM. IEEE Trans. Infor. Theory 28, 129-137 (1982).

63. Steinhardt, P. J., Nelson, D. R. \& Ronchetti, M. Bond-orientational order in liquids and glasses. Phys. Rev. B 28, 784-805 (1983).

64. Lechner, W. \& Dellago, C. Accurate determination of crystal structures based on averaged local bond order parameters. J. Chem. Phys. 129, 114707 (2008).

65. Yu, H. B. \& Samwer, K. Atomic mechanism of internal friction in a model metallic glass. Phys. Rev. B 90, 144201 (2014).

66. Pryor, A., Ophus, C. \& Miao, J. A streaming multi-GPU implementation of image simulation algorithms for scanning transmission electron microscopy. Adv. Struct. Chem. Imag. 3, 15 (2017).

67. Warren, B. E. X-ray diffraction (New York: Dover Publications Inc. 1990).

68. Cowley, J. M. X-Ray Measurement of order in single crystals of Cu3Au. J. Appl. Phys. 21, 24-29 (1950).

69. Lee, C. Y. An algorithm for path connections and its applications. IEEE Trans. Electron. Comput. EC-10, 346-365 (1961).

70. Larsen, P. M., Schmidt, S. \& Schiøtz, J. Robust structural identification via polyhedral template matching. Model. Simul. Mater. Sci. Eng. 24, 055007 (2016).

71. Tran, D. T., Svensson, G., \& Tai, C. W. SUePDF: a program to obtain quantitative pair distribution functions from electron diffraction data. J. Appl. Crystallogr. 50, 304-312 (2017). 


\section{Figures}
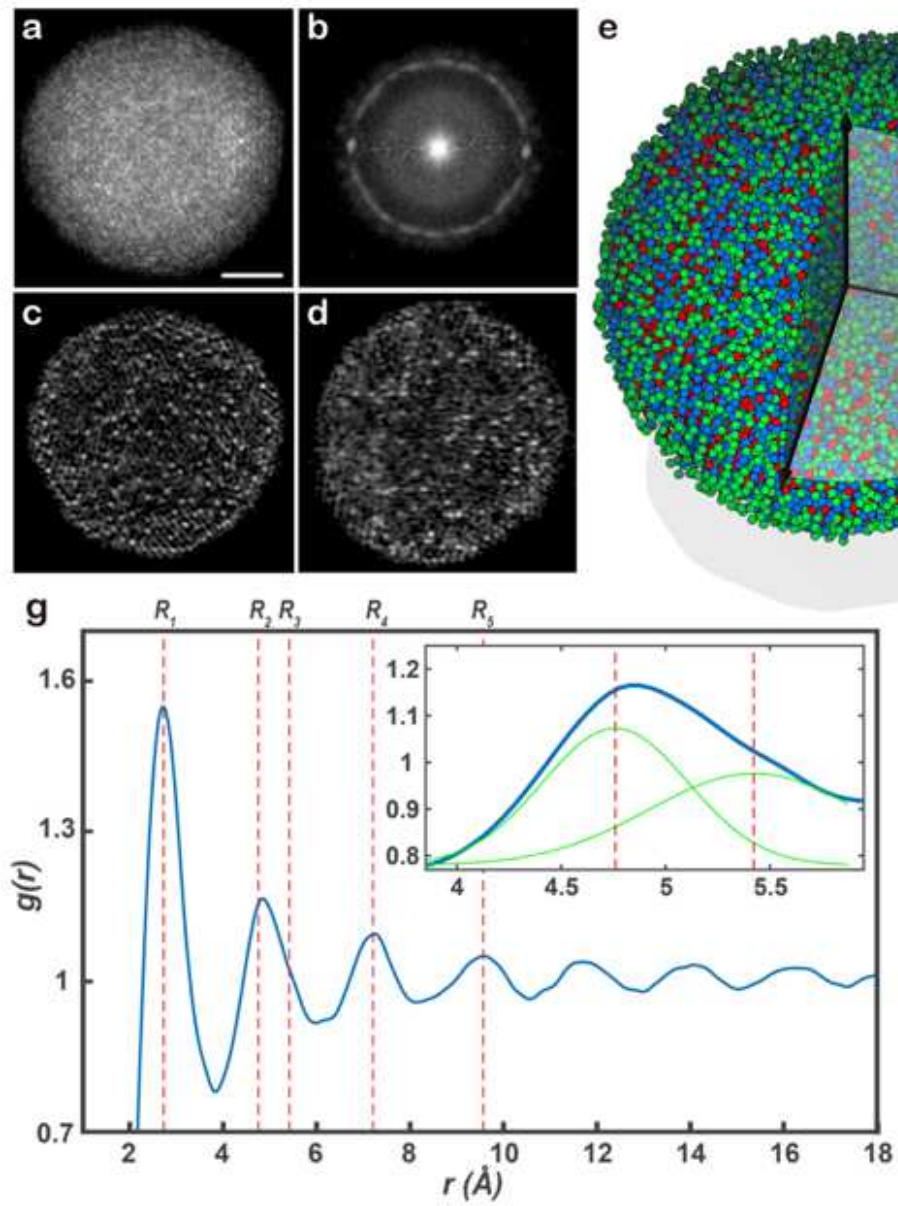
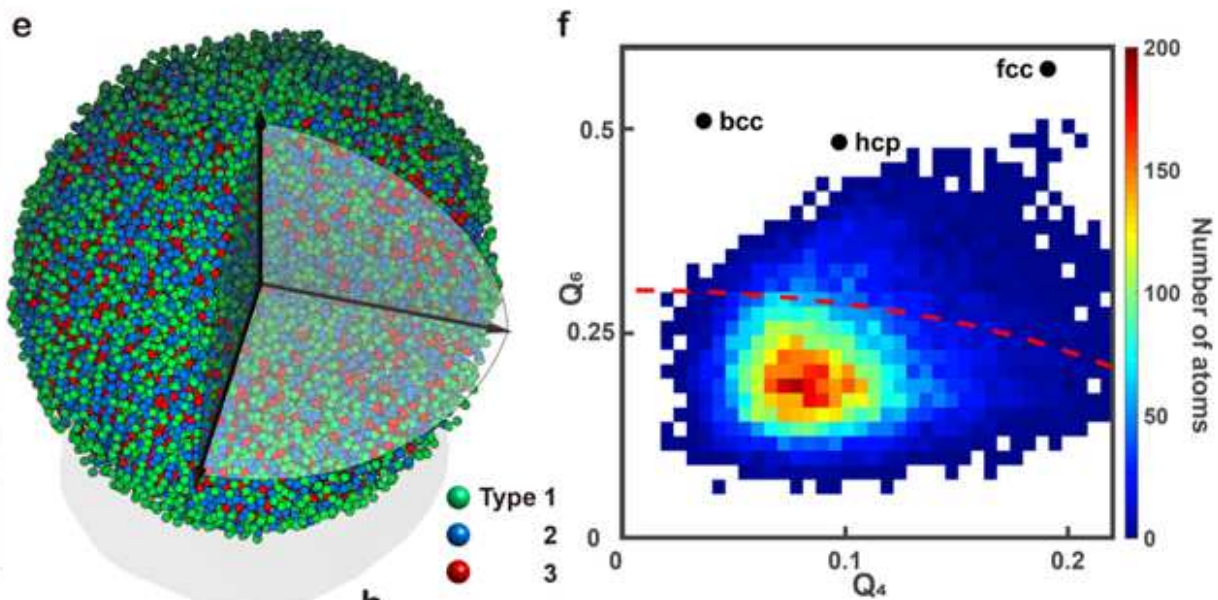

h

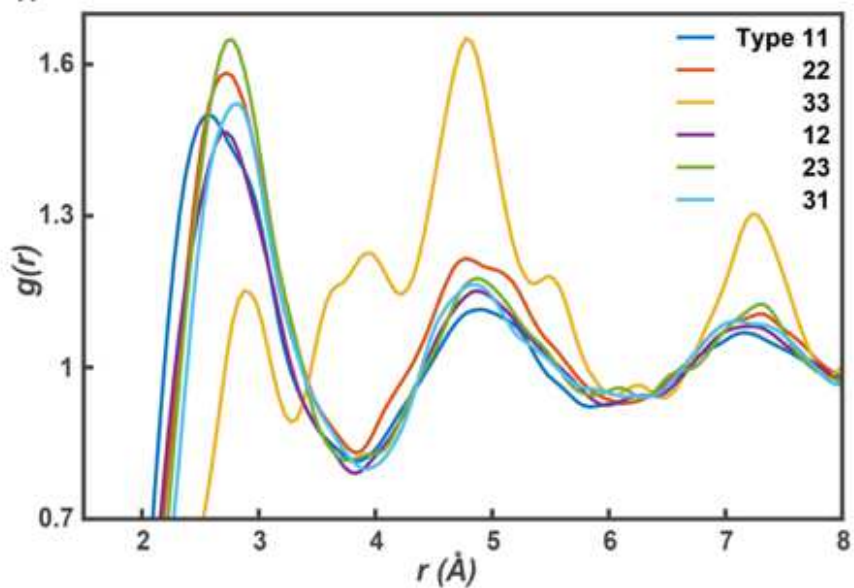

\section{Figure 1}

Determining the $3 \mathrm{D}$ atomic structure of a multi-component metallic glass with $\mathrm{AET}$. a, A representative experimental image, where some crystalline features are visible. b, Average diffraction pattern obtained from 55 experimental images (Extended Data Fig. 3a), showing the amorphous halo. c, d, Two 2.4-Å-thick slices of the 3D reconstruction in the xy-and yz-plane, respectively, where the majority of type 3 atoms (bright dots) are distributed in the second coordination shell. e, Experimental 3D atomic model of the metallic glass nanoparticle. $f$, The local BOO parameters of all the atoms in the nanoparticle. Based on the criterion of the normalized BOO parameter $<0.5$ (the dashed red curve), $84.54 \%$ of the total atoms are disordered. $g$, The RDF of the disordered atoms with the first, second, third, fourth and fifth peak positions (R1, R2, R3, R4 and R5) at 2.73, 4.76, 5.42, 7.22 and $9.57 \AA$, respectively, which are in good agreement with those in the RDF directly derived from the average diffraction pattern (Extended Data Fig. 3c). The inset shows the second-peak splitting with a Gaussian fit. h, The partial PDFs between type 1, 2 and 3 atoms, consisting of 6 pairs - type 11, 12, 13, 22, 23 and 33 atoms. The partial PDF for the type 33 atoms (the yellow curve) shows a unique feature with a higher second peak than the first peak. 
a

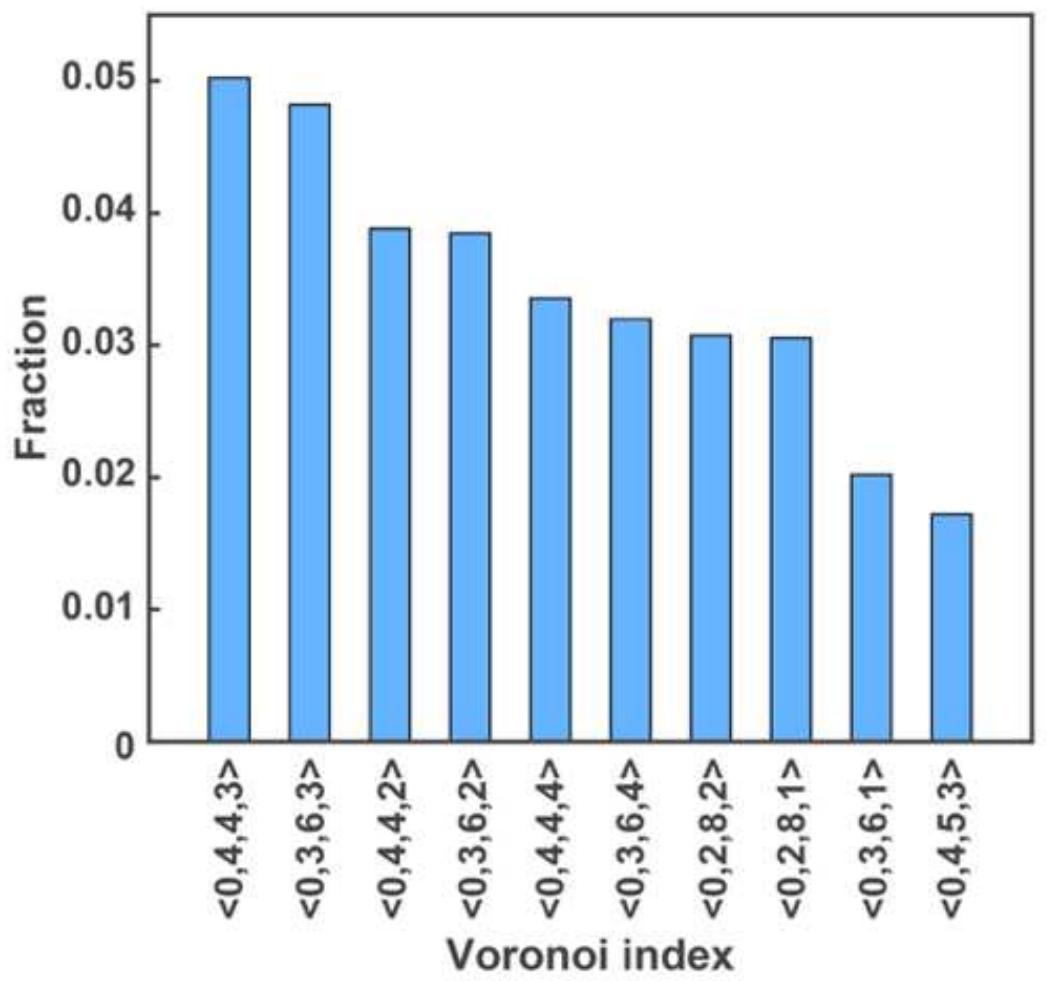

C

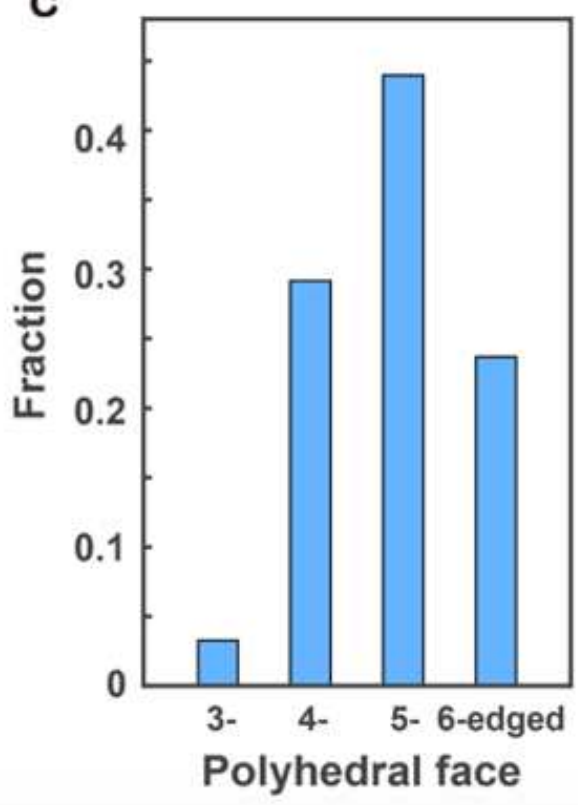

d b

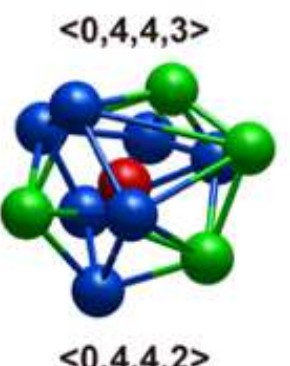

$\langle 0,4,4,2\rangle$

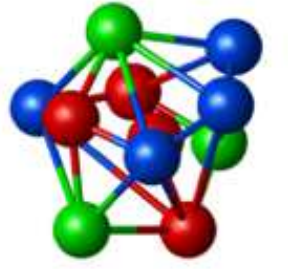

$<0,4,4,4>$

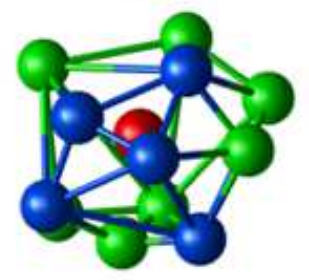

Type 1

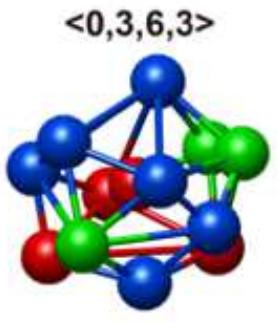

$<0,3,6,2\rangle$

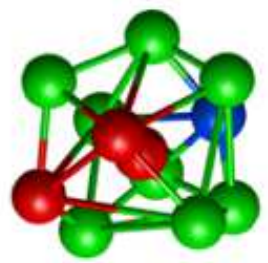

$\langle 0,0,12,0\rangle$

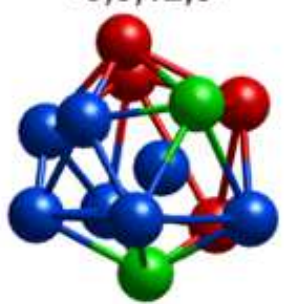

Type 2 Type 3

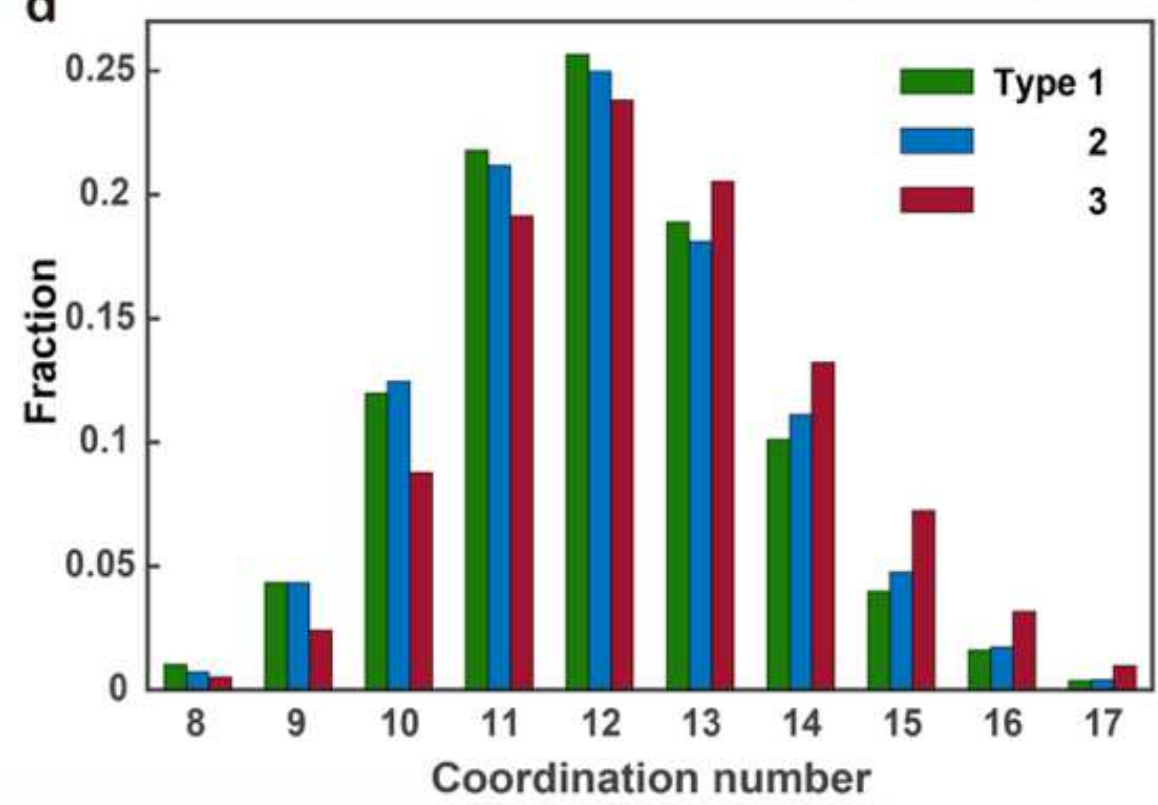

Figure 2

The short-range order of the metallic glass nanoparticle. a, Ten most abundant Voronoi polyhedra in the nanoparticle. b, Six representative Voronoi polyhedra, where $\langle 0,4,4,3\rangle,\langle 0,3,6,3\rangle,\langle 0,4,4,2\rangle$ and $\langle 0,3,6,2\rangle$ are the four highest fraction Voronoi indices, $\langle 0,4,4,4\rangle$ shows a severely distorted polyhedron, and $\langle 0,0,12,0\rangle$ represents an icosahedron. c, The 3-, 4-, 5- and 6-edged face distribution of all the Voronoi polyhedra, where the 5-edged faces are the most abundant (43.91\%). $d$, The coordination number (CN) distribution for type 1, 2 and 3 atoms. 

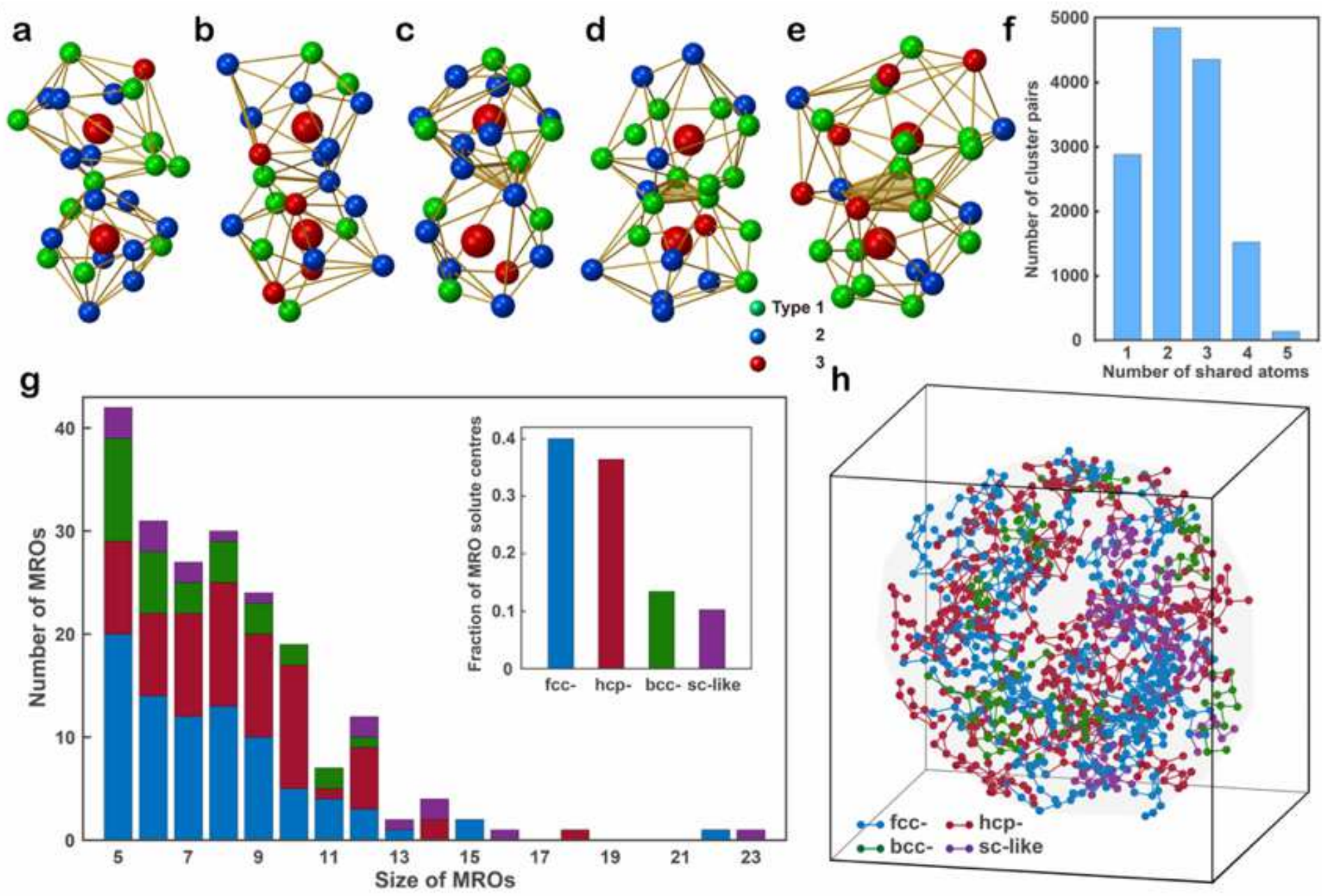

\section{Figure 3}

The connectivity and distribution of the MRO in the metallic glass nanoparticle. a-e, Representative pairs of the solute-centred clusters that are connected with each other by sharing one, two, three, four and five atoms, respectively, where the central atom of each cluster is indicated by a large red sphere. $\mathrm{f}$, Statistical distribution of the number of the solute-centred cluster pairs, which share from one to five atoms. $\mathrm{g}$, Histogram of the four types of MRO - fcc- (in blue), hcp- (in red), bcc- (in green) and sc-like (in purple) - as a function of the size (i.e. the number of solute centres). The total number of fcc-, hcp-, bcc- and sc-like MRO is $85,71,31$ and 17, respectively. The inset shows the fraction of the solute centre atoms in the four types of MRO. h, Distribution of the four types of the MRO with eight solute centre atoms or more, where the centre region lacks of large MRO. 

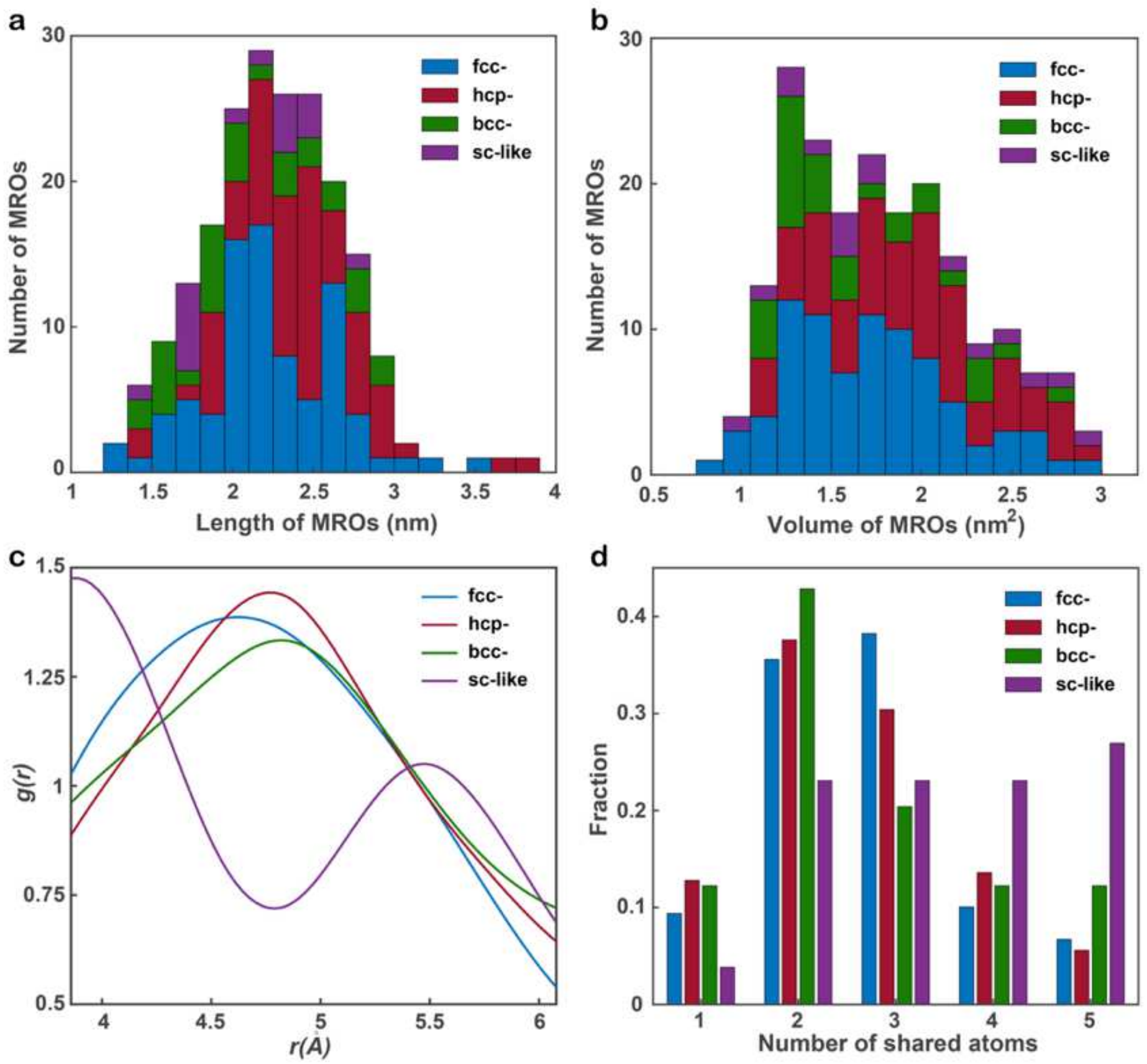

Figure 4

Quantitative characterization of the MRO. The length (a) and volume (b) distribution of the four types of the MRO in the metallic glass nanoparticle, where the length was measured along the longest direction of each MRO. c, Partial PDFs of the fcc-, hcp-, bcc- and sc-like solute centres in the metallic glass nanoparticle, where the maximum peak positions are located at $4.62,4.77,4.82$ and $3.88 \AA$, respectively. Compared with the other three partial PDFs, the partial PDF of the sc-like solute centres (the purple curve) shows two peaks with the ratio of the second to the first peak position about $\sqrt{ } 2$. $d$, Distribution of sharing one, two, three, four and five atoms between neighbouring solute-centred clusters for the four types of the MRO. 

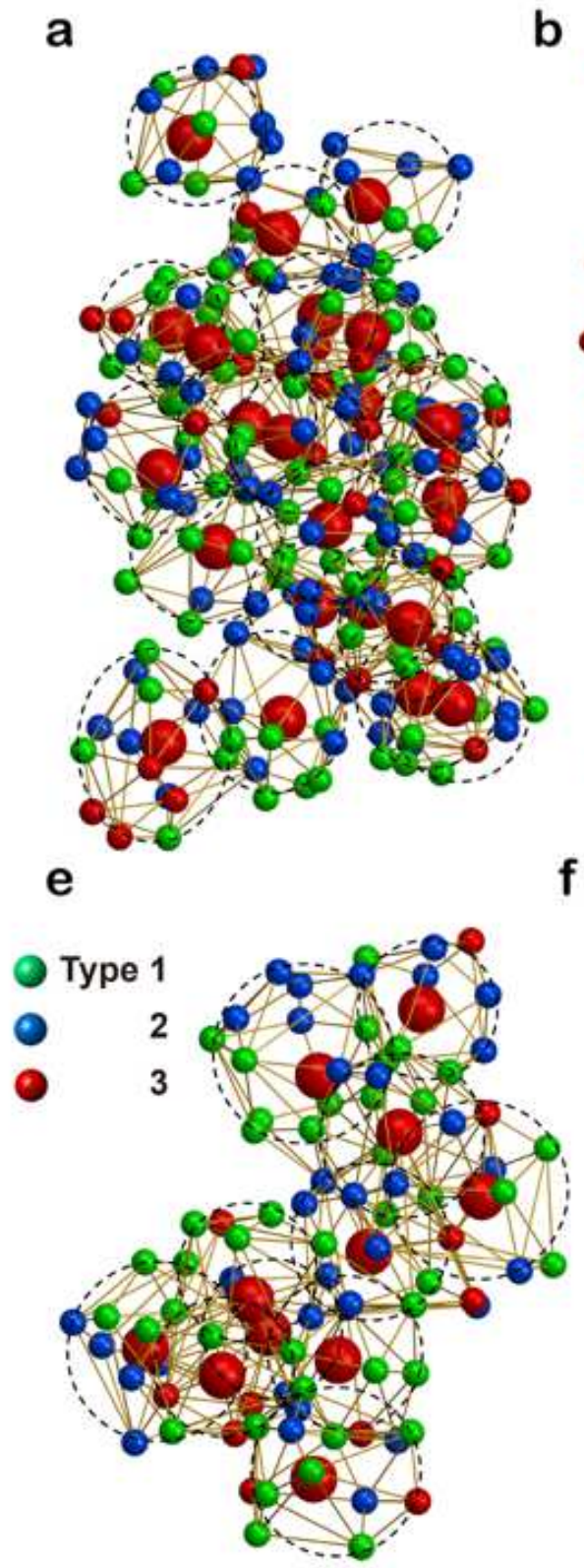

b

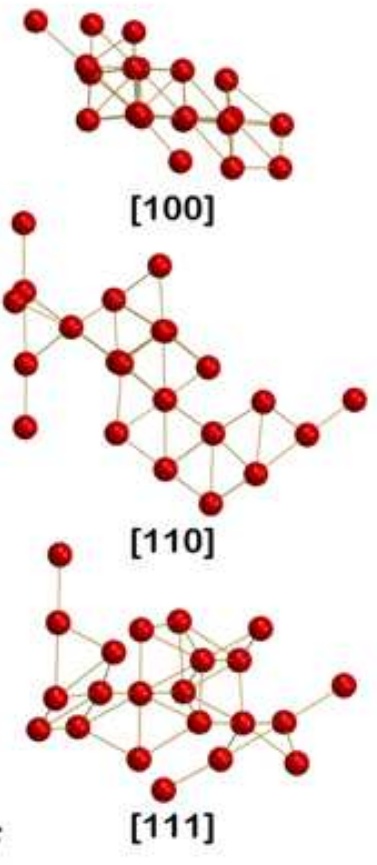

C
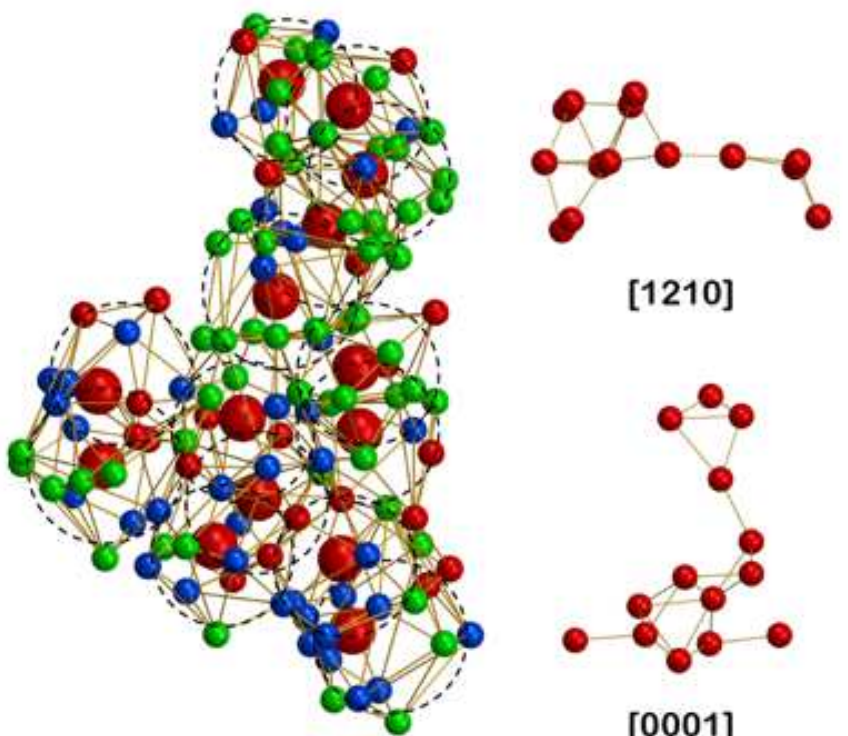

g

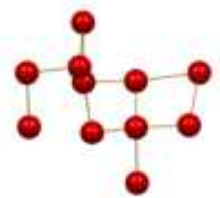

[100]

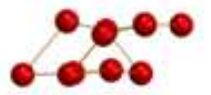

[110]

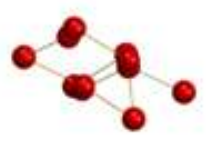

[111]
[1210]

d

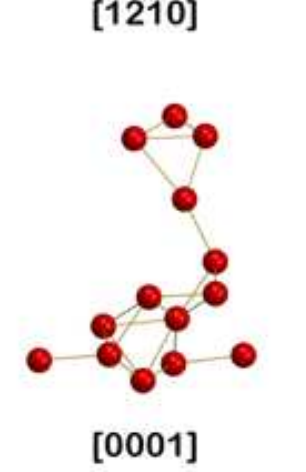

h
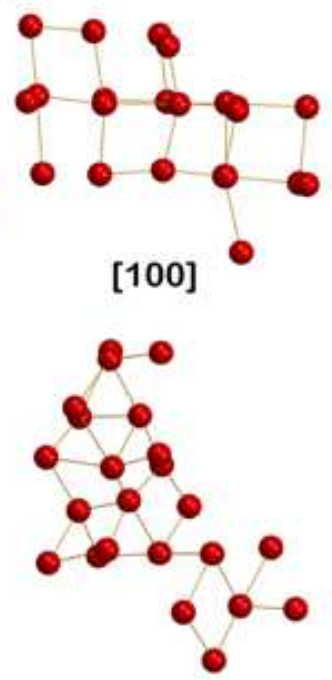

[111]

Figure 5

3D atomic packing of four representative MRO. Representative fcc- (a), hcp- (c), bcc- (e) and sc-like (g) MRO, consisting of 22, 14, 11 and 23 solute centres (large red spheres), respectively, where the solutecentred clusters (dashed circles) show only translational but no orientational order. To better visualize the crystal-like MRO, the solvent atoms are removed and the solute centres are orientated along the fcc (b), hcp (d), bcc (f) and sc (h) zone axes, showing that the 3D shapes of the MRO are anisotropic and the networks significantly deviate from perfect crystal lattices.

\section{Supplementary Files}


This is a list of supplementary files associated with this preprint. Click to download.

- Video1.mov

- Video2.mov

- Video3.mov

- Video4.mov

- Supplementarylnfo.docx

- MiaoExtendedDatarev.docx 